\title{
RIEMANNIAN INVARIANTS THAT CHARACTERIZE ROTATIONAL SYMMETRIES OF THE STANDARD SPHERE
}

\author{
MASAYUKI AINO
}

\begin{abstract}
Inspired by the Lichnerowicz-Obata theorem for the first eigenvalue of the Laplacian, we define a new family of invariants $\left\{\Omega_{k}(g)\right\}$ for closed Riemannian manifolds. The value of $\Omega_{k}(g)$ sharply reflects the spherical part of the manifold. Indeed, $\Omega_{1}(g)$ and $\Omega_{2}(g)$ characterize the standard sphere.
\end{abstract}

\section{Contents}

1. Introduction

2. The definition of $\Lambda_{k}(S)$ and elementary properties

2.1. Preliminaries

2.2. The definition and elementary properties of $\Lambda_{k}(S)$

2.3. Other properties of $\Lambda_{k}(S)$

3. Main properties of $\Omega_{k}$

3.1. The proofs of Theorem A and Theorem C

3.2. The proof of Theorem B

3.3. An estimate in the presence of a parallel $p$-form

3.4. Riemannian manifolds whose value of $\Omega_{1}$ is close to $\frac{n-1}{n}$

4. Computation and examples

4.1. The product of Einstein manifolds

4.2. The case of Heisenberg manifolds

Appendix A. The proof of Lemma 2.5

References

\section{INTRODUCTION}

In this paper we introduce a new family of Riemannian invariants that characterizes the standard sphere. Indeed, we define $\Omega_{1} \geq \Omega_{2} \geq \cdots \rightarrow 0$ for closed Riemannian manifolds, and show that $\Omega_{1}$ and $\Omega_{2}$ characterize the standard sphere. Before explaining our result in detail, we provide historical backgrounds.

Let $(M, g)$ be a closed Riemannian manifold of dimension $n$ and $\operatorname{Spec}(M, g)=$ $\left\{0=\lambda_{0}<\lambda_{1} \leq \lambda_{2} \leq \cdots \rightarrow \infty\right\}$ the set of eigenvalues of the Laplacian $\Delta=$ $-g^{i j} \nabla_{i} \nabla_{j}$ acting on $C^{\infty}(M)$. We can construct a complete orthonormal system $\left\{\psi_{i}\right\}$ in $L^{2}(M)$ of eigenfunctions of $\Delta$ :

$$
\Delta \psi_{i}=\lambda_{i} \psi_{i} .
$$

Eigenvalues characterize the standard sphere in some cases. For example, Tanno [10, Theorem B] showed that, for $n \leq 6$, $\operatorname{Spec}\left(S^{n}(c)\right)$ completely characterizes the standard $n$-dimensional sphere $S^{n}(c)$ of radius $c$, i.e., if $\operatorname{Spec}(M, g)=\operatorname{Spec}\left(S^{n}(c)\right)$, 
then $(M, g)$ is isometric to $S^{n}(c)$. Under the condition Ric $\geq r g$ for a positive constant $r>0$, Lichnerowicz [5] showed

$$
\lambda_{1} \geq \frac{n}{n-1} r
$$

and Obata showed the equality holds if and only if $(M, g)$ is isometric to the standard sphere of radius $\sqrt{(n-1) / r}$. In the proof, the following theorem $[6$, Theorem A] plays an important role.

Theorem 1.1. Let $c$ be a positive constant. If $(M, g)$ admits a non-constant function $u \in C^{\infty}(M)$ with $\nabla^{2} u=-c^{2} u g$, then $(M, g)$ is isometric to $S^{n}(1 / c)$.

We emphasize that this theorem needs no assumption about Ricci curvature. Similarly, Tashiro [8, Lemma2.2] showed the following theorem.

Theorem 1.2. If $(M, g)$ admits a non-constant function $u \in C^{\infty}(M)$ with $\nabla^{2} u=$ $-\frac{\Delta u}{n} g$, then $(M, g)$ is conformal to $S^{n}(1)$.

This theorem plays an important role in Theorem A below. We review Tashiro's work in section 3 .

In this paper, inspired by these results, we define non-negative real numbers $\Omega_{1}(g) \geq \Omega_{2}(g) \geq \Omega_{3}(g) \geq \cdots \rightarrow 0$ for a closed Riemannian manifold $(M, g)$, and study their properties. The value of $\Omega_{1}(g)$ is defined by

$$
\Omega_{1}(g)=\sup \left\{\int_{M} \operatorname{Ric}(\nabla v, \nabla v) d \mu_{g}: v \in L_{2}^{2}(M) \text { and }\|\Delta v\|_{L^{2}}=1\right\} .
$$

We define $\left\{\Omega_{k}(g)\right\}_{k \geq 2}$ in Definition 1 and Definition 2. If $\Omega_{1}(g)>0$, then we have $\Omega_{k}(g)>0$ for any $k \in \mathbb{Z}_{>0}$, and there exists a non-constant function $v_{k} \in C^{\infty}(M)$ such that $\Delta^{2} v_{k}=\frac{1}{\Omega_{k}(g)} \nabla^{*}\left(\operatorname{Ric}\left(\nabla v_{k}, \cdot\right)\right)$. We have the following two theorems:

Theorem A . For any closed $n$-dimensional Riemannian manifold $(M, g)$, we have $\Omega_{1}(g) \leq \frac{n-1}{n}$. If $\Omega_{1}(g)=\frac{n-1}{n}$, then $(M, g)$ is conformal to the standard sphere.

Theorem B . Let $(M, g)$ be a closed Riemannian manifold of dimension $n$. If $\Omega_{1}(g)=\Omega_{2}(g)=\frac{n-1}{n}$, then $(M, g)$ is isometric to the standard sphere of a certain radius.

Consequently, $\Omega_{1}(g)$ and $\Omega_{2}(g)$ characterize the $n$-dimensional standard sphere. Roughly speaking, $\Omega_{1}(g)=\frac{n-1}{n}$ means that $(M, g)$ has a rotational symmetry. Moreover, $\Omega_{2}(g)=\frac{n-1}{n}$ means that $(M, g)$ has another rotation axis. Theorem $\mathrm{B}$ results from the fact that no Riemannian manifold except for the standard sphere has two distinct rotation axes (for the explicit statement, see Lemma 3.3).

The relationship between Theorem A and Lichnerowicz inequality is the following:

Theorem C . If there exists a constant $r>0$ such that Ric $\geq r g$, then

$$
\lambda_{1} \geq \frac{r}{\Omega_{1}(g)} \geq \frac{n}{n-1} r .
$$

In Theorem 3.5, we see that the value of $\Omega_{1}(g)$ sharply reflects the spherical part of the manifold. In section 4 , we give some computation and examples.

In the next section, we define a family of non-negative numbers $\left\{\Lambda_{k}(S)\right\}_{k \in \mathbb{Z}_{>0}}$ for a closed Riemannian manifold $(M, g)$ and a symmetric tensor $S$ of type $(0,2)$ on $M$. In section 3 , we specialize the case when $S=\operatorname{Ric}_{g}$, and $\Lambda_{k}\left(\operatorname{Ric}_{g}\right)$ recovers $\Omega_{k}(g)$ above.

Acknowledgments. I am grateful to my supervisor, Professor Shinichiroh Matsuo, for his useful comments and advice. 


\section{The Definition of $\Lambda_{k}(S)$ AND ELEMENTARY PROPERTIES}

\subsection{Preliminaries.}

Notation 1. Let $(M, g)$ be a closed Riemannian manifold of dimension $n$. $H$ denotes the closed subspace of $L_{2}^{2}(M)$ defined by

$$
H=H_{(M, g)}=\left\{v \in L_{2}^{2}(M): \int_{M} v d \mu_{g}=0\right\}
$$

with an inner product

$$
\langle u, v\rangle_{H}=\int_{M}(\Delta u, \Delta v) d \mu_{g} \quad(u, v \in H) .
$$

The norm induced by this inner product is equivalent to the norm induced by the standard inner product on $L_{2}^{2}(M)$. Note that $\left.\operatorname{Ker} \Delta\right|_{H}=\{0\}$, and so there exists a constant $C>0$ such that $\|v\|_{L_{2}^{2}} \leq C\|\Delta v\|_{L^{2}}$ for all $v \in H$. This is what is called the elliptic estimate.

Notation 2. Let $S$ be a symmetric tensor of type $(0,2)$ on $M$. We define a functional $\Lambda_{S}: H \backslash\{0\} \rightarrow \mathbb{R}$ by

$$
\Lambda_{S}(v)=\frac{\int_{M} S(\nabla v, \nabla v) d \mu_{g}}{\|\Delta v\|_{L^{2}}^{2}}
$$

By the elliptic estimate, we have $\sup _{v \in H \backslash\{0\}} \Lambda_{S}(v)<\infty$. Note that, for each $v \in L_{2}^{2}(M)$, there exists a constant $c \in \mathbb{R}$ such that $v-c \in H$, and so

$$
\sup _{v \in H \backslash\{0\}} \Lambda_{S}(v)=\sup \left\{\frac{\int_{M} S(\nabla v, \nabla v) d \mu_{g}}{\|\Delta v\|_{L^{2}}^{2}}: v \in L_{2}^{2}(M) \text { and } v \text { is not a constant }\right\} \text {. }
$$

Lemma 2.1. Let $F \neq\{0\}$ be a closed subspace of $H$. If $\sup _{v \in F \backslash\{0\}} \Lambda_{S}(v)>0$, then there exists a function $u \in F \backslash\{0\}$ such that $\sup _{v \in F \backslash\{0\}} \Lambda_{S}(v)=\Lambda_{S}(u)$.

Proof. Put $\Lambda=\sup _{v \in F \backslash\{0\}} \Lambda_{S}(v)$. Let $\left\{u_{k}\right\}$ be a sequence of $F$ such that $\Lambda=$ $\lim _{k \rightarrow \infty} \Lambda_{S}\left(u_{k}\right)$ and $\left\|\Delta u_{k}\right\|_{L^{2}}=1$. By the elliptic estimate, there exists a constant $C>0$ such that $\left\|u_{k}\right\|_{L_{2}^{2}} \leq C$ for all $k$. Thus, we can take a subsequence of $\left\{u_{k}\right\}$ (denote it again by $\left\{u_{k}\right\}$ ) and $u \in F$ such that

$$
\begin{aligned}
& u_{k} \rightarrow u\left(\text { strongly in } L_{1}^{2}(M)\right), \\
& u_{k} \rightarrow u(\text { weakly in } F) .
\end{aligned}
$$

Then,

$$
\begin{aligned}
& \Lambda-\Lambda_{S}\left(u_{k}\right) \\
= & \Lambda \int_{M}\left(\Delta u_{k}, \Delta u_{k}\right) d \mu_{g}-\int_{M} S\left(\nabla u_{k}, \nabla u_{k}\right) d \mu_{g} \\
= & \Lambda \int_{M}\left(\Delta u+\Delta\left(u_{k}-u\right), \Delta u+\Delta\left(u_{k}-u\right)\right) d \mu_{g} \\
& \quad-\int_{M} S(\nabla u, \nabla u) d \mu_{g}+\epsilon_{k} \\
= & \Lambda\|\Delta u\|_{L^{2}}^{2}+2 \Lambda \int_{M}\left(\Delta\left(u_{k}-u\right), \Delta u\right) d \mu_{g}+\Lambda\left\|\Delta\left(u_{k}-u\right)\right\|_{L^{2}}^{2} \\
& \quad-\int_{M} S(\nabla u, \nabla u) d \mu_{g}+\epsilon_{k} \\
\geq & 2 \Lambda \int_{M}\left(\Delta\left(u_{k}-u\right), \Delta u\right) d \mu_{g}+\Lambda\left\|\Delta\left(u_{k}-u\right)\right\|_{L^{2}}^{2}+\epsilon_{k},
\end{aligned}
$$


where we put $\epsilon_{k}=\int_{M} S(\nabla u, \nabla u) d \mu_{g}-\int_{M} S\left(\nabla u_{k}, \nabla u_{k}\right) d \mu_{g}$. In the last line, we have used $\int_{M} S(\nabla u, \nabla u) d \mu_{g} \leq \Lambda\|\Delta u\|_{L^{2}}^{2}$. By (1), we have

$$
\begin{aligned}
& \Lambda\left\|\Delta\left(u_{k}-u\right)\right\|_{L^{2}}^{2} \\
\leq & \Lambda-\Lambda_{S}\left(u_{k}\right)-2 \Lambda \int_{M}\left(\Delta\left(u_{k}-u\right), \Delta u\right) d \mu_{g}-\epsilon_{k} .
\end{aligned}
$$

The right hand side of (2) converges to 0 as $k \rightarrow \infty$. Since we assumed $\Lambda>0$, we get

$$
\lim \left\|\Delta\left(u_{k}-u\right)\right\|_{L^{2}}=0 .
$$

This implies that $u_{k}$ converges to $u$ strongly in $H$, and so $\Lambda=\Lambda_{S}(u)$.

Now, we consider the case when $F=H$.

Lemma 2.2. Put $\Lambda_{1}(S)=\sup _{v \in H \backslash\{0\}} \Lambda_{S}(v)$. If $\Lambda_{1}(S)>0$, for $u \in H \backslash\{0\}$, the following two conditions are equivalent:

(a) $\Lambda_{1}(S)=\Lambda_{S}(u)$

(b) $\Delta^{2} u=\frac{1}{\Lambda_{1}(S)} \nabla^{*}(S(\nabla u, \cdot))$.

In particular, if $u$ satisfies $\Lambda_{1}(S)=\Lambda_{S}(u)$, then $u$ is smooth.

Proof. We first prove (a) $\Rightarrow$ (b). Suppose that $u \in H \backslash\{0\}$ satisfies $\Lambda_{1}(S)=\Lambda_{S}(u)$, i.e.,

$$
\Lambda_{1}(S)=\sup _{v \in H \backslash\{0\}} \frac{\int_{M} S(\nabla v, \nabla v) d \mu_{g}}{\|\Delta v\|_{L^{2}}^{2}}=\frac{\int_{M} S(\nabla u, \nabla u) d \mu_{g}}{\|\Delta u\|_{L^{2}}^{2}} .
$$

Then, for any $v \in L_{2}^{2}(M)$, we have

$$
\begin{gathered}
\Lambda_{1}(S) \int_{M}(\Delta(u+t v), \Delta(u+t v)) d \mu_{g}-\int_{M} S(\nabla(u+t v), \nabla(u+t v)) d \mu_{g} \geq 0 \quad(t \in \mathbb{R}), \\
\Lambda_{1}(S) \int_{M}(\Delta u, \Delta u) d \mu_{g}-\int_{M} S(\nabla u, \nabla u) d \mu_{g}=0 .
\end{gathered}
$$

Therefore,

$$
\begin{aligned}
0 & =\left.\frac{d}{d t}\right|_{t=0}\left(\Lambda_{1}(S) \int_{M}(\Delta(u+t v), \Delta(u+t v)) d \mu_{g}\right. \\
& \left.\quad-\int_{M} S(\nabla(u+t v), \nabla(u+t v)) d \mu_{g}\right) \\
= & 2 \Lambda_{1}(S) \int_{M}(\Delta u, \Delta v) d \mu_{g}-2 \int_{M} S(\nabla u, \nabla v) d \mu_{g} \\
= & 2 \Lambda_{1}(S) \int_{M}\left(\Delta^{2} u-\frac{1}{\Lambda_{1}(S)} \nabla^{*}(S(\nabla u, \cdot)), v\right) d \mu_{g} .
\end{aligned}
$$

Since the pairing $L_{-2}^{2}(M) \times L_{2}^{2}(M) \rightarrow \mathbb{R}$ is non-degenerate, we conclude $\Delta^{2} u-$ $\frac{1}{\Lambda_{1}(S)} \nabla^{*}(S(\nabla u, \cdot))=0$.

We next prove (b) $\Rightarrow$ (a). Suppose that $\Delta^{2} u=\frac{1}{\Lambda_{1}(S)} \nabla^{*}(S(\nabla u, \cdot))$. Then we have,

$$
\int_{M} S(\nabla u, \nabla u) d \mu_{g}=\int_{M}\left(\nabla^{*}(S(\nabla u, \cdot)), u\right) d \mu_{g}=\Lambda_{1}(S) \int_{M}(\Delta u, \Delta u) d \mu_{g} .
$$

Therefore, we get $\Lambda_{1}(S)=\frac{\int_{M} S(\nabla u, \nabla u) d \mu_{g}}{\|\Delta u\|_{L^{2}}^{2}}=\Lambda_{S}(u)$.

Next we consider the sign of $\sup _{v \in F \backslash\{0\}} \Lambda_{S}(v)$.

Lemma 2.3. For any symmetric tensor $S$ of type $(0,2)$ and any infinite dimensional closed subspace $F \subset H$, we have $\sup _{v \in F \backslash\{0\}} \Lambda_{S}(v) \geq 0$. Moreover, if $S \leq 0$, then $\sup _{v \in F \backslash\{0\}} \Lambda_{S}(v)=0$ holds. 
Proof. Since $M$ is compact, there exists a constant $c>0$ such that $S \geq-c g$. Let

$$
0=\lambda_{0}<\lambda_{1} \leq \lambda_{2} \leq \cdots \rightarrow \infty
$$

be the eigenvalues of the Laplacian $\Delta$ and $\left\{\psi_{i}\right\}$ a complete orthonormal system in $L^{2}(M)$ of the eigenfunctions of $\Delta$ :

$$
\Delta \psi_{i}=\lambda_{i} \psi_{i}
$$

Since $F \subset H$ is infinite dimensional, for any $k=0,1,2, \ldots$, we can take $v_{k} \in$ $F \backslash\{0\}$ that is orthogonal to $\psi_{0}, \ldots, \psi_{k}$ in $L^{2}(M)$. Then,

$$
\begin{aligned}
\int_{M} S\left(\nabla v_{k}, \nabla v_{k}\right) d \mu_{g} & \geq-c \int_{M}\left(\nabla v_{k}, \nabla v_{k}\right) d \mu_{g} \\
& \geq-\frac{c}{\lambda_{k+1}}\left\|\Delta v_{k}\right\|_{L^{2}}^{2} .
\end{aligned}
$$

Therefore, $\sup _{v \in F \backslash\{0\}} \Lambda_{S}(v) \geq \Lambda_{S}\left(v_{k}\right) \geq-\frac{c}{\lambda_{k+1}}$. The right hand side converges to 0 as $k \rightarrow \infty$, and so we get $\sup _{v \in F \backslash\{0\}} \Lambda_{S}(v) \geq 0$.

Moreover, if $S \leq 0$, we have $\Lambda_{S}(v) \leq 0$ for any $v \in F \backslash\{0\}$. Therefore, $\sup _{v \in F \backslash\{0\}} \Lambda_{S}(v) \leq 0$. Thus, we get $\sup _{v \in F \backslash\{0\}} \Lambda_{S}(v)=0$.

In the case when $F=H$, the converse is also true:

Lemma 2.4. If $\sup _{v \in H \backslash\{0\}} \Lambda_{S}(v)=0$, then we have $S \leq 0$.

Moreover, we can show the following:

Lemma 2.5. If $S \leq 0$ does not hold, for any positive integer $k \in \mathbb{N}$, there exists a $k$-dimensional subspace $V_{k}$ of $C^{\infty}(M) \cap H$ that satisfies the following: $\Lambda_{S}(v)>0$ holds for any $v \in V_{k} \backslash\{0\}$.

Putting $k=1$ in Lemma 2.5, we get Lemma 2.4. See Appendix for the proof of Lemma 2.5.

2.2. The definition and elementary properties of $\Lambda_{k}(S)$. Let $(M, g)$ be a closed Riemannian manifold of dimension $n$ and $S$ a symmetric tensor of type $(0,2)$ on $M$. We define $\Lambda_{1}(S) \geq \Lambda_{2}(S) \geq \cdots$.

Definition 1. We put $\Lambda_{1}(S)=\sup _{v \in H \backslash\{0\}} \Lambda_{S}(v)$. If $\Lambda_{1}(S)=0$, we define $\Lambda_{1}(S)=$ $\Lambda_{2}(S)=\Lambda_{3}(S)=\cdots=0$. If $\Lambda_{1}(S)>0$, we can take a function $v_{1} \in H$ such that $\Lambda_{S}\left(v_{1}\right)=\Lambda_{1}(S)$ and $\left\|\Delta v_{1}\right\|_{L^{2}}=1$ by Lemma 2.1. We define $\Lambda_{k}(S)$ and $v_{k}$ inductively as follows. Suppose that we have chosen $\Lambda_{1}(S), \cdots, \Lambda_{k}(S)>0$ and $v_{1}, \cdots, v_{k} \in H$. We put

$$
\Lambda_{k+1}(S)=\sup \left\{\Lambda_{S}(v): v \in\left\langle v_{1}, \cdots, v_{k}\right\rangle^{\perp} \backslash\{0\}\right\},
$$

where $\left\langle v_{1}, \cdots, v_{k}\right\rangle^{\perp}$ denotes the orthogonal complement of $\operatorname{Span}_{\mathbb{R}}\left\{v_{1}, \cdots, v_{k}\right\}$ in $H$. We have $\Lambda_{k+1}(S)>0$ by Lemma 2.3 and Lemma 2.5. therefore, we can take $v_{k+1} \in\left\langle v_{1}, \cdots, v_{k}\right\rangle^{\perp} \backslash\{0\}$ such that $\Lambda_{k+1}(S)=\Lambda_{S}\left(v_{k+1}\right)$ and $\left\|\Delta v_{k+1}\right\|_{L^{2}}=1$ by Lemma 2.1 Inductively, we define $\Lambda_{1}(S) \geq \Lambda_{2}(S) \geq \cdots>0$ and $v_{1}, v_{2}, \cdots \in H$. We call $v_{k}$ the associated function to $\Lambda_{k}(S)$.

We can prove each $v_{k}$ is smooth. In fact, we show the following:

Proposition 2.6. If $\Lambda_{1}(S)>0$, then we have $\Delta^{2} v_{i}=\frac{1}{\Lambda_{i}(S)} \nabla^{*}\left(S\left(\nabla v_{i}, \cdot\right)\right)$ for each $i=1,2, \ldots$.

To prove Proposition 2.6, we show the following lemma. 
Lemma 2.7. Take a real number $\Lambda \in \mathbb{R}$ and a function $v \in H$. Suppose that $\Lambda \Delta^{2} v=\nabla^{*}(S(\nabla v, \cdot))$ holds. Then, we have

$$
\int_{M} S(\nabla v, \nabla u) d \mu_{g}=0
$$

for any function $u \in H$ with $\langle u, v\rangle_{H}=0$.

Proof. For any function $u \in H$ with $\langle u, v\rangle_{H}=0$, we have

$$
\begin{aligned}
\int_{M} S(\nabla v, \nabla u) d \mu_{g} & =\int_{M}\left(\nabla^{*}(S(\nabla v, \cdot)), u\right) d \mu_{g} \\
& =\Lambda \int_{M}\left(\Delta^{2} v, u\right) d \mu_{g} \\
& =\Lambda \int_{M}(\Delta v, \Delta u) d \mu_{g}=0 .
\end{aligned}
$$

Proof of Proposition [2.6. We show the proposition by induction on $i$. We have shown the proposition for $i=1$ in Lemma 2.1. Suppose that we have shown the proposition for $i=1, \ldots, k$. Then, for any $v \in\left\langle v_{1}, \ldots, v_{k}\right\rangle^{\perp}$, similarly to Lemma 2.2, we have

$$
\int_{M}\left(\Delta^{2} v_{k+1}-\frac{1}{\Lambda_{k+1}(S)} \nabla^{*}\left(S\left(\nabla v_{k+1}, \cdot\right)\right), v\right) d \mu_{g}=0 .
$$

For each $v_{i}(i=1, \cdots, k)$, we have $\left\langle v_{k+1}, v_{i}\right\rangle_{H}=0$. Therefore, by Lemma 2.7 .

$$
\int_{M}\left(\nabla^{*}\left(S\left(\nabla v_{k+1}, \cdot\right)\right), v_{i}\right) d \mu_{g}=0
$$

Thus,

$$
\int_{M}\left(\Delta^{2} v_{k+1}-\frac{1}{\Lambda_{k+1}(S)} \nabla^{*}\left(S\left(\nabla v_{k+1}, \cdot\right)\right), v_{i}\right) d \mu_{g}=0 .
$$

Consequently, (3) holds for any $v \in L_{2}^{2}(M)$. This implies the proposition for $i=$ $k+1$.

We introduce some elementary properties of $\Lambda_{k}(S)$.

Proposition 2.8. For any symmetric tensor $S$ of type $(0,2)$, we have $\lim _{k \rightarrow \infty} \Lambda_{k}(S)=$ 0 .

Proof. We assume $\Lambda_{1}(S)>0$ (otherwise the lemma is trivial).

We put $\Lambda=\lim _{k \rightarrow \infty} \Lambda_{k}(S)$. Let $H^{+}(S)=\overline{\bigoplus_{k=1}^{\infty} \mathbb{R} v_{k}}$ be the closure of $\bigoplus_{k=1}^{\infty} \mathbb{R} v_{k}$ in $H$. For any $v \in H^{+}(S) \backslash\{0\}$, we have

$$
v=\lim _{l \rightarrow \infty} \sum_{k=1}^{l}\left\langle v, v_{k}\right\rangle v_{k} \quad(\text { strongly in } H) .
$$

Therefore, by Lemma 2.7

$$
\begin{aligned}
\Lambda_{S}(v) & =\frac{\int_{M} S(\nabla v, \nabla v) d \mu_{g}}{\|\Delta v\|_{L^{2}}^{2}} \\
& =\frac{\sum\left\langle v, v_{k}\right\rangle^{2} \int_{M} S\left(\nabla v_{k}, \nabla v_{k}\right) d \mu_{g}}{\sum\left\langle v, v_{k}\right\rangle^{2}\left\|\Delta v_{k}\right\|_{L^{2}}^{2}} \\
& \geq \frac{\sum\left\langle v, v_{k}\right\rangle^{2} \Lambda\left\|\Delta v_{k}\right\|_{L^{2}}^{2}}{\sum\left\langle v, v_{k}\right\rangle^{2}\left\|\Delta v_{k}\right\|_{L^{2}}^{2}}=\Lambda .
\end{aligned}
$$


Then, for any $v \in H^{+}(S) \backslash\{0\}$, we have $\Lambda_{-S}(v) \leq-\Lambda$, and $\operatorname{sos} \sup _{v \in H^{+}(S) \backslash\{0\}} \Lambda_{-S}(v) \leq$ $-\Lambda$. By Lemma 2.3. we have $\sup _{v \in H^{+}(S) \backslash\{0\}} \Lambda_{-S}(v) \geq 0$; therefore, $-\Lambda \geq 0$. However, we have $\Lambda \geq 0$. Thus, $\Lambda=0$ holds.

Lemma 2.9. Take real numbers $\Lambda, \bar{\Lambda} \in \mathbb{R}$ and functions $v, \bar{v} \in H$. If $\Lambda \neq \bar{\Lambda}$ and

$$
\begin{aligned}
& \Lambda \Delta^{2} v=\nabla^{*}(S(\nabla v, \cdot)) \\
& \bar{\Lambda} \Delta^{2} \bar{v}=\nabla^{*}(S(\nabla \bar{v}, \cdot)),
\end{aligned}
$$

then $\langle v, \bar{v}\rangle_{H}=0$.

Proof.

$$
\begin{aligned}
\Lambda \int_{M}(\Delta v, \Delta \bar{v}) d \mu_{g} & =\int_{M}\left(\nabla^{*}(S(\nabla v, \cdot)), \bar{v}\right) d \mu_{g} \\
& =\int_{M}\left(v, \nabla^{*}(S(\nabla \bar{v}, \cdot))\right) d \mu_{g} \\
& =\bar{\Lambda} \int_{M}(\Delta v, \Delta \bar{v}) d \mu_{g} .
\end{aligned}
$$

Thus, we get $\int_{M}(\Delta v, \Delta \bar{v}) d \mu_{g}=0$.

We define $\Lambda_{-i}(S)=-\Lambda_{i}(-S)$. If $-\Lambda_{-1}(S)>0$, we define $v_{-i}$ to be the associated function to $\Lambda_{-i}(S)$, i.e., $v_{-1}, v_{-2}, \cdots$ are orthonormal in $H$, and $\Delta^{2} v_{-i}=$ $\frac{1}{\Lambda_{-i}(S)} \nabla^{*}\left(S\left(\nabla v_{-i}, \cdot\right)\right)$ holds. We define

$$
\begin{aligned}
& H^{+}(S)=\overline{\bigoplus_{k=1}^{\infty} \mathbb{R} v_{k}}, \\
& H^{-}(S)=H^{+}(-S)=\overline{\bigoplus_{k=1}^{\infty} \mathbb{R} v_{-k}},
\end{aligned}
$$

where the overline means the closure in $H$ (if $\Lambda_{1}(S)=0$, then we define $H^{+}(S)=$ $\{0\})$. Then, $H^{+}(S)$ and $H^{-}(S)$ are orthogonal to each other in $H$ by Lemma 2.9. Let $H^{0}(S)$ be the orthogonal complement of $H^{+}(S) \oplus H^{-}(S)$ in $H$. We can characterize the element of $H^{0}(S)$ by the following lemma.

Proposition 2.10. For each $v \in H$ the following two conditions are equivalent:

(a) $v \in H^{0}(S)$,

(b) $\nabla^{*}(S(\nabla v, \cdot))=0$.

Proof. We first prove $(\mathrm{a}) \Rightarrow(\mathrm{b})$. Take $v \in H^{0}(S)$. We assume $v \neq 0$ (otherwise the lemma is trivial). For any $k=1,2, \ldots$, we have $v \in\left\langle v_{1}, \cdots, v_{k}\right\rangle^{\perp}$. Thus, $\Lambda_{S}(v) \leq \Lambda_{k+1}(S)$. Taking the limit, we get $\Lambda_{S}(v) \leq 0$ by Proposition 2.8. Similarly, we have $\Lambda_{-S}(v) \leq 0$; therefore, $\Lambda_{S}(v)=0$. Thus, for any $w \in H^{0}(S)$ and $t \in \mathbb{R}$, we have $\int_{M} S(\nabla(v+t w), \nabla(v+t w)) d \mu_{g}=0$. Therefore, we get

$$
\int_{M} S(\nabla v, \nabla w) d \mu_{g}=0
$$

By Lemma 2.7, for any $k=1,2, \ldots$,

$$
\int_{M} S\left(\nabla v, \nabla v_{k}\right) d \mu_{g}=\int_{M} S\left(\nabla v, \nabla v_{-k}\right) d \mu_{g}=0 .
$$

This implies that the equation (4) holds for all $w \in H$. Therefore, we get $\nabla^{*}(S(\nabla v, \cdot))=$ 0 .

We next prove $(\mathrm{b}) \Rightarrow(\mathrm{a})$. Suppose $\nabla^{*}(S(\nabla v, \cdot))=0$. Then, $v$ is orthogonal to $H^{+}(S)$ and $H^{-}(S)$ by Lemma 2.9. Thus, we get (a).

Next, we characterize $v_{k}$

Lemma 2.11. Take $u \in H \backslash\{0\}$. Suppose that there exists a constant $a \in \mathbb{R}$ such that $\Delta^{2} u=a \nabla^{*}(S(\nabla u, \cdot))$. Then, there exists a non-zero integer $k \in \mathbb{Z} \backslash\{0\}$ such that $a=\frac{1}{\Lambda_{k}(S)}$ and $u \in \operatorname{Span}_{\mathbb{R}}\left\{v_{i}: \Lambda_{S}\left(v_{i}\right)=\Lambda_{k}(S)\right\}$. 
Proof. Since $\Delta^{2} u \neq 0$, we have $a \neq 0$. Then, $u$ is orthogonal to $H^{0}(S)$ and $v_{i}$ for $i \in \mathbb{Z} \backslash\{0\}$ such that $\frac{1}{\Lambda_{i}(S)} \neq a$ by Lemma 2.9. Therefore, there exists a non-zero integer $k \in \mathbb{Z} \backslash\{0\}$ such that $\frac{1}{\Lambda_{k}(S)}=a$ and $u \in \operatorname{Span}_{\mathbb{R}}\left\{v_{i}: \Lambda_{S}\left(v_{i}\right)=\Lambda_{k}(S)\right\}$.

Lemma 2.11 immediately implies the following two corollaries.

Corollary 2.12. Suppose that $\left\{u_{l}\right\}_{l=1}^{\infty}$ is a complete orthonormal system in $H$, and, for each $l \in \mathbb{Z}_{>0}$, there exists $c_{l} \in \mathbb{R}$ such that $c_{l} \Delta^{2} u_{l}=\nabla^{*}\left(S\left(\nabla u_{l}, \cdot\right)\right)$. Then,

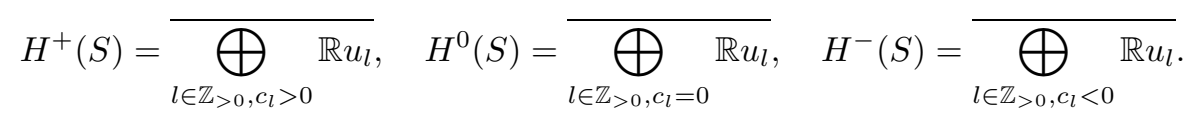

Moreover, if $\Lambda_{1}(S)>0$, we have

$$
\left\{\Lambda_{k}(S): k \in \mathbb{Z}_{>0}\right\}=\left\{c_{l}: l \in \mathbb{Z}_{>0} \text { and } c_{l}>0\right\} .
$$

Corollary 2.13. Let $0<\lambda_{1} \leq \lambda_{2} \leq \cdots \rightarrow \infty$ be the eigenvalues of the Laplacian. Then, we have $\Lambda_{k}(g)=\frac{1}{\lambda_{k}}$ for all $k \in \mathbb{Z}_{>0}$.

2.3. Other properties of $\Lambda_{k}(S)$. We consider the value of $\Lambda_{1}$ of the product of Riemannian manifolds. Let $\left(M_{i}, g_{i}\right)(i=1,2)$ be $n_{i}$-dimensional compact Riemannian manifolds, and $S_{i}$ symmetric tensors of type $(0,2)$ on $M_{i}$. We denote the projections by $\pi_{i}: M_{1} \times M_{2} \rightarrow M_{i}$. Then, we have $T\left(M_{1} \times M_{2}\right)=\pi_{1}^{*} T M_{1} \bigoplus \pi_{2}^{*} T M_{2}$. We consider the product metric $g=g_{1}+g_{2}$ on $M=M_{1} \times M_{2}$. We define a symmetric tensor of type $(0,2)$ on $M$ by $S=S_{1} \oplus S_{2}$. Then, we have the following proposition.

Proposition 2.14. We have $\Lambda_{1}(S)=\max \left\{\Lambda_{1}\left(S_{1}\right), \Lambda_{1}\left(S_{2}\right)\right\}$.

Proof. Let $\left\{\lambda_{i}\right\}$ (resp. $\left\{\lambda_{k}^{\prime}\right\}$ ) be the eigenvalues and $\left\{\psi_{i}\right\}$ (resp. $\left\{\psi_{k}^{\prime}\right\}$ ) the eigenfunctions of the Laplacian of $\left(M_{1}, g_{1}\right)$ (resp. $\left.\left(M_{2}, g_{2}\right)\right)$. For any $u \in C^{\infty}(M)$, we have the following decomposition:

$$
u(x, y)=\sum a_{i k} \psi_{i}(x) \psi_{k}^{\prime}(y)=\sum b_{k}(x) \psi_{k}^{\prime}(y)=\sum c_{i}(y) \psi_{i}(x) .
$$

Therefore,

$$
\begin{aligned}
S(\nabla u, \nabla u) & =\sum a_{i k} a_{j l}\left(S_{1}\left(\nabla^{g_{1}} \psi_{i}, \nabla^{g_{1}} \psi_{j}\right) \psi_{k}^{\prime} \psi_{l}^{\prime}+\psi_{i} \psi_{j} S_{2}\left(\nabla^{g_{2}} \psi_{k}^{\prime}, \nabla^{g_{2}} \psi_{l}^{\prime}\right)\right) \\
& =\sum S_{1}\left(\nabla^{g_{1}} b_{k}, \nabla^{g_{1}} b_{l}\right) \psi_{k}^{\prime} \psi_{l}^{\prime}+\sum \psi_{i} \psi_{j} S_{2}\left(\nabla^{g_{2}} c_{i}, \nabla^{g_{2}} c_{j}\right) .
\end{aligned}
$$

By the integration, we have

$$
\begin{aligned}
& \int_{M} S(\nabla u, \nabla u) d \mu_{g} \\
= & \sum_{k} \int_{M_{1}} S_{1}\left(\nabla^{g_{1}} b_{k}, \nabla^{g_{1}} b_{k}\right) d \mu_{g_{1}}+\sum_{i} \int_{M_{2}} S_{2}\left(\nabla^{g_{2}} c_{i}, \nabla^{g_{2}} c_{i}\right) d \mu_{g_{2}} \\
\leq & \Lambda_{1}\left(S_{1}\right) \sum_{k} \int_{M_{1}}\left(\Delta^{g_{1}} b_{k}, \Delta^{g_{1}} b_{k}\right) d \mu_{g_{1}}+\Lambda_{1}\left(S_{2}\right) \sum_{i} \int_{M_{2}}\left(\Delta^{g_{2}} c_{i}, \Delta^{g_{2}} c_{i}\right) d \mu_{g_{2}} .
\end{aligned}
$$

We have $b_{k}(x)=\sum a_{i k} \psi_{i}(x)$, and so

$$
\int_{M_{1}}\left(\Delta^{g_{1}} b_{k}, \Delta^{g_{1}} b_{k}\right) d \mu_{g_{1}}=\sum_{i} a_{i k}^{2} \lambda_{i}^{2}
$$

Similarly, we have

$$
\int_{M_{2}}\left(\Delta^{g_{2}} c_{i}, \Delta^{g_{2}} c_{i}\right) d \mu_{g_{2}}=\sum_{k} a_{i k}^{2} \lambda_{k}^{\prime 2} .
$$


By (5)-(7) and $\Delta\left(\psi_{i} \psi_{k}^{\prime}\right)=\left(\lambda_{i}+\lambda_{k}^{\prime}\right) \psi_{i} \psi_{k}^{\prime}$, we get

$$
\begin{aligned}
\int_{M}(\Delta u, \Delta u) d \mu_{g} & =\sum_{i, k} a_{i k}^{2}\left(\lambda_{i}+\lambda_{k}^{\prime}\right)^{2} \\
& \geq \sum_{i, k} a_{i k}^{2} \lambda_{i}^{2}+\sum_{i k} a_{i k}^{2} \lambda_{k}^{\prime 2} \\
& =\sum_{k} \int_{M_{1}}\left(\Delta^{g_{1}} b_{k}, \Delta^{g_{1}} b_{k}\right) d \mu_{g_{1}}+\sum_{i} \int_{M_{2}}\left(\Delta^{g_{2}} c_{i}, \Delta^{g_{2}} c_{i}\right) d \mu_{g_{2}} \\
& \left.\geq \frac{1}{\max \left\{\Lambda_{1}\left(S_{1}\right), \Lambda_{1}\left(S_{2}\right)\right\}}\left(\Lambda_{1}\left(S_{1}\right) \sum_{k} \int_{M_{1}}\left(\Delta^{g_{1}} b_{k}, \Delta^{g_{1}} b_{k}\right) d \mu_{g_{1}}\right) \sum_{i} \int_{M_{2}}\left(\Delta^{g_{2}} c_{i}, \Delta^{g_{2}} c_{i}\right) d \mu_{g_{2}}\right) \\
& \geq \frac{1}{\max \left\{\Lambda_{1}\left(S_{1}\right), \Lambda_{1}\left(S_{2}\right)\right\}} \int_{M} S(\nabla u, \nabla u) d \mu_{g} .
\end{aligned}
$$

Therefore,

$$
\Lambda_{1}(S) \leq \max \left\{\Lambda_{1}\left(S_{1}\right), \Lambda_{1}\left(S_{2}\right)\right\}
$$

Next, we prove that the equality holds. Suppose that $\Lambda_{1}\left(S_{1}\right) \geq \Lambda_{1}\left(S_{2}\right)$. Moreover, we assume $\Lambda_{1}\left(S_{1}\right)>0$ (otherwise the proposition is trivial). Take a nonconstant function $v_{1} \in C^{\infty}\left(M_{1}\right)$ such that $\Lambda_{1}\left(S_{1}\right)=\Lambda_{S_{1}}\left(v_{1}\right)$. We regard $v_{1}$ as a smooth function on $M ; v_{1} \in C^{\infty}(M)$. Then,

$$
\Lambda_{1}(S) \geq \Lambda_{S}\left(v_{1}\right)=\Lambda_{S_{1}}\left(v_{1}\right)=\Lambda_{1}\left(S_{1}\right)=\max \left\{\Lambda_{1}\left(S_{1}\right), \Lambda_{1}\left(S_{2}\right)\right\}
$$

By (8) and (9), we get $\Lambda_{1}(S)=\max \left\{\Lambda_{1}\left(S_{1}\right), \Lambda_{1}\left(S_{2}\right)\right\}$.

We give a useful formula for $\Lambda_{i}(S)$.

Proposition 2.15. Let $(M, g)$ be a closed Riemannian manifold and $S$ a symmetric tensor of type $(0,2)$ on $M$. We have

$$
\Lambda_{k}(S)=\sup _{L_{k} \subset H}\left[\inf _{v \in L_{k} \backslash\{0\}} \Lambda_{S}(v)\right]
$$

for any positive integer $k \in \mathbb{Z}_{>0}$, where $L_{k}$ varies over all $k$-dimensional subspaces of $H$.

Proof. If $\Lambda_{1}(S)=0$, we have $S \leq 0$. Similarly to Lemma 2.3, we can show

$$
\sup _{L_{k} \subset H}\left[\inf _{v \in L_{k} \backslash\{0\}} \Lambda_{S}(v)\right]=0
$$

for any $k \in \mathbb{Z}_{>0}$, and so the proposition holds.

Suppose that $\Lambda_{1}(S)>0$. Let $\left\{v_{k}\right\}_{\mathbb{Z}>0}$ be the associated functions to $\left\{\Lambda_{k}(S)\right\}_{\mathbb{Z}>0}$. Take a positive integer $k \in \mathbb{Z}_{>0}$. Let $L_{k}$ be a $k$-dimensional subspace of $H$. Then, there exists a function $v \in L_{k} \backslash\{0\}$ such that $v$ is orthogonal to $v_{1}, \ldots, v_{k-1}$. By definition of $\Lambda_{k}(S)$, we have $\Lambda_{S}(v) \leq \Lambda_{k}(S)$. Therefore, $\inf _{v \in L_{k} \backslash\{0\}} \Lambda_{S}(v) \leq \Lambda_{k}(S)$ holds. Thus,

$$
\Lambda_{k}(S) \geq \sup _{L_{k} \subset H}\left[\inf _{v \in L_{k} \backslash\{0\}} \Lambda_{S}(v)\right] .
$$

Let us show the equality holds. We put $L_{k}^{0}=\operatorname{Span}_{\mathbb{R}}\left\{v_{1}, \cdots, v_{k}\right\}$. Then, we have $\Lambda_{k}(S)=\inf _{v \in L_{k}^{0} \backslash\{0\}} \Lambda_{S}(v)$. This implies

$$
\Lambda_{k}(S)=\sup _{L_{k} \subset H}\left[\inf _{v \in L_{k} \backslash\{0\}} \Lambda_{S}(v)\right] .
$$


Corollary 2.16. Let $S$ and $T$ be symmetric tensors of type $(0,2)$ on $M$. If $S \geq T$, then we have $\Lambda_{k}(S) \geq \Lambda_{k}(T)$ for each $k \in \mathbb{Z} \backslash\{0\}$. Moreover, if $\Lambda_{k}(S)=\Lambda_{k}(T)$ for all $k \in \mathbb{Z} \backslash\{0\}$, then $S=T$ holds.

Proof. Take a positive integer $k \in \mathbb{Z}_{>0}$. We have

$$
\inf _{v \in L_{k} \backslash\{0\}} \Lambda_{S}(v) \geq \inf _{v \in L_{k} \backslash\{0\}} \Lambda_{T}(v)
$$

for any $k$-dimensional subspace $L_{k}$ of $H$. Thus, we get $\Lambda_{k}(S) \geq \Lambda_{k}(T)$ by Proposition 2.15. Similarly, we have $\Lambda_{k}(-T) \geq \Lambda_{k}(-S)$; therefore, $\Lambda_{-k}(S) \geq \Lambda_{-k}(T)$.

Suppose that $\Lambda_{k}(S)=\Lambda_{k}(T)$ holds for all $k \in \mathbb{Z} \backslash\{0\}$. If $\Lambda_{1}(T)=0$, then $H^{+}(S)=H^{+}(T)=\{0\}$. Assume that $\Lambda_{1}(T)>0$ and take the associated functions $\left\{v_{k}\right\}_{\mathbb{Z}_{>0}}$ to $\left\{\Lambda_{k}(T)\right\}_{\mathbb{Z}_{>0}}$ for $T$. Take a positive integer $l \in \mathbb{Z}_{>0}$ such that $\Lambda_{l-1}(T)<$ $\Lambda_{l}(T)$ (put $\Lambda_{0}(T)=0$ ). Suppose that $\Lambda_{l}(T)=\Lambda_{l+K}(T)$ for $K \in \mathbb{Z}_{\geq 0}$. For each $i=0, \ldots, K$, we put $L_{l, i}^{0}=\operatorname{Span}_{\mathbb{R}}\left\{v_{1}, \cdots, v_{l-1}, v_{l+i}\right\}$. By Proposition 2.15, we have

$$
\Lambda_{l+i}(S)=\Lambda_{l}(S) \geq \inf _{v \in L_{l, i}^{0} \backslash\{0\}} \Lambda_{S}(v) .
$$

Take $v \in L_{l, i}^{0} \backslash\{0\}$ that attains the infimum of the right hand side. Then, we have $\Lambda_{l+i}(S) \geq \Lambda_{S}(v) \geq \Lambda_{T}(v) \geq \Lambda_{l+i}(T)$. By $\Lambda_{l+i}(S)=\Lambda_{l+i}(T)$, we have $v=v_{l+i}$ and $\Lambda_{l+i}(S)=\Lambda_{S}\left(v_{l+i}\right)$. Thus, $\left\{v_{k}\right\}_{\mathbb{Z}_{>0}}$ is the family of associated functions to $\left\{\Lambda_{k}(S)\right\}_{\mathbb{Z}_{>0}}$ for $S$, and so $\int_{M} S(\nabla v, \nabla v) d \mu_{g}=\int_{M} T(\nabla v, \nabla v) d \mu_{g}$ for any $v \in$ $H^{+}(S)=H^{+}(T)$. Similarly, we have $H^{-}(S)=H^{-}(T)$ and $\int_{M} S(\nabla v, \nabla v) d \mu_{g}=$ $\int_{M} T(\nabla v, \nabla v) d \mu_{g}$ for any $v \in H^{-}(S)=H^{-}(T)$. Consequently, we have $H^{0}(S)=$ $H^{0}(T)$ and $\int_{M} S(\nabla v, \nabla v) d \mu_{g}=\int_{M} T(\nabla v, \nabla v) d \mu_{g}$ for any $v \in H$. This and Lemma 2.4 imply $S=T$.

\section{MAIN PROPERTIES OF $\Omega_{k}$}

3.1. The proofs of Theorem A and Theorem C. In this section we consider the case when $S=\operatorname{Ric}_{g}$.

Definition 2. We put $\Omega_{k}(g)=\Lambda_{k}\left(\operatorname{Ric}_{g}\right)$ for a closed Riemannian manifold $(M, g)$.

In this case, the following Bochner formula plays an important role.

Proposition 3.1. Let $(M, g)$ be a Riemannian manifold and $\omega$ a 1-form on $M$. Then, we have

$$
\Delta \omega=\nabla^{*} \nabla \omega+\operatorname{Ric}\left(\omega^{\sharp}, \cdot\right),
$$

where $\omega^{\sharp}$ denotes a vector field such that $g\left(\omega^{\sharp}, Y\right)=\omega(Y)$ for any vector field $Y$.

By the Bochner formula, we have the following theorem.

Theorem A . For any closed n-dimensional Riemannian manifold $(M, g)$, we have $\Omega_{1}(g) \leq \frac{n-1}{n}$. If $\Omega_{1}(g)=\frac{n-1}{n}$, then $(M, g)$ is conformal to the standard sphere.

Proof. For any function $v \in C^{\infty}(M)$, we have $\Delta v=-\operatorname{tr} \nabla^{2} v$; therefore,

$$
0 \leq\left(\nabla^{2} v+\frac{1}{n} \Delta v g, \nabla^{2} v+\frac{1}{n} \Delta v g\right)=\left(\nabla^{2} v, \nabla^{2} v\right)-\frac{1}{n}(\Delta v, \Delta v) .
$$

Thus, we have

$$
\|\Delta v\|_{L^{2}}^{2} \leq n\left\|\nabla^{2} v\right\|_{L^{2}}^{2} .
$$


By the Bochner formula and (10), we have

$$
\begin{aligned}
\int_{M} \operatorname{Ric}(\nabla v, \nabla v) d \mu_{g} & =\int_{M}(\Delta d v, d v) d \mu_{g}-\int_{M}\left(\nabla^{*} \nabla d v, d v\right) d \mu_{g} \\
& =\int_{M}(d \Delta v, d v) d \mu_{g}-\int_{M}\left(\nabla^{2} v, \nabla^{2} v\right) d \mu_{g} \\
& =\int_{M}(\Delta v, \Delta v) d \mu_{g}-\int_{M}\left(\nabla^{2} v, \nabla^{2} v\right) d \mu_{g} \\
& \leq \frac{n-1}{n} \int_{M}(\Delta v, \Delta v) d \mu_{g}
\end{aligned}
$$

Consequently, we get $\Lambda_{\mathrm{Ric}}(v) \leq \frac{n-1}{n}$ for any non-constant function $v \in C^{\infty}(M)$. Therefore, we get $\Omega_{1}(g) \leq \frac{n-1}{n}$.

Suppose that $\Omega_{1}(g)=\frac{n-1}{n}$. Then, there exists a function $v_{1} \in H$ such that $\Lambda_{\text {Ric }}\left(v_{1}\right)=\frac{n-1}{n}$, and so $\left\|\Delta v_{1}\right\|_{L^{2}}^{2}=n\left\|\nabla^{2} v_{1}\right\|_{L^{2}}^{2}$. Therefore, we have $\nabla^{2} v_{1}+$ $\frac{1}{n} \Delta v_{1} g=0$. Thus, Theorem 1.2 implies the theorem.

Remark 3.1. We have

$$
\|\Delta v\|_{L^{2}}^{2} \leq \frac{1}{1-\Omega_{1}(g)}\left\|\nabla^{2} v\right\|_{L^{2}}^{2}
$$

for any $v \in C^{\infty}(M)$.

Proposition 3.2. Let $0<\lambda_{1} \leq \lambda_{2} \leq \cdots \rightarrow \infty$ be the eigenvalues of the Laplacian. If there exists a constant $r>0$ such that Ric $\geq r g$, then we have

$$
\lambda_{k} \geq \frac{r}{\Omega_{k}(g)}
$$

for any $k=1,2, \ldots$ Moreover, if $\lambda_{k}=\frac{r}{\Omega_{k}(g)}$ holds for all $k=1,2, \ldots$, then Ric $=$ rg holds.

Proof. By Corollary 2.13, we have $\Lambda_{k}(r g)=\frac{r}{\lambda_{k}}$. Thus, Corollary 2.16] implies the proposition.

Putting $k=1$, we get the following.

Theorem C . If there exists a constant $r>0$ such that Ric $\geq$ rg, then

$$
\lambda_{1} \geq \frac{r}{\Omega_{1}(g)} \geq \frac{n}{n-1} r
$$

Let us consider the behavior of $\Omega_{k}(g)$ under the homothetic transformation. Let $(M, g)$ be a closed Riemannian manifold and $a$ a positive constant. Then, for any $u \in C^{\infty}(M)$ we have $\nabla^{a^{2} g} u=\frac{1}{a^{2}} \nabla^{g} u, \Delta^{a^{2} g} u=\frac{1}{a^{2}} \Delta^{g} u, \operatorname{Ric}_{a^{2} g}=\operatorname{Ric}_{g}$ and $d \mu_{a^{2} g}=a^{n} d \mu_{g}$. Thus, if $u$ is a non-constant function, we have

$$
\frac{\int_{M} \operatorname{Ric}_{a^{2} g}\left(\nabla^{a^{2} g} u, \nabla^{a^{2} g} u\right) d \mu_{a^{2} g}}{\int_{M}\left(\Delta^{a^{2} g} u, \Delta^{a^{2} g} u\right) d \mu_{a^{2} g}}=\frac{\int_{M} \operatorname{Ric}_{g}\left(\nabla^{g} u, \nabla^{g} u\right) d \mu_{g}}{\int_{M}\left(\Delta^{g} u, \Delta^{g} u\right) d \mu_{g}} .
$$

Therefore, we have $\Omega_{k}\left(a^{2} g\right)=\Omega_{k}(g)$ for any $k \in \mathbb{Z} \backslash\{0\}$.

On the other hand, $\lambda_{k}\left(a^{2} g\right)=\frac{1}{a^{2}} \lambda_{k}(g)$ holds. Based on these, we give an example of non-Einstein Riemannian manifold such that Ric $\geq r g$ and $\lambda_{1}=\frac{r}{\Omega_{1}(g)}$ for some positive constant $r>0$.

Example 3.1. Let $\left(M_{i}, g_{i}\right)(i=1,2)$ be closed Einstein manifolds of dimension $n_{i}$ such that $\operatorname{Ric}_{g_{i}}=g_{i}$ holds. We assume that $\Omega_{1}\left(g_{1}\right) \geq \Omega_{1}\left(g_{2}\right)$. For each $r>1$, we consider the metric $G_{r}=r^{2} g_{1}+g_{2}$ on the product manifold $M=M_{1} \times M_{2}$. If $r$ is large enough, we have $\lambda_{1}\left(G_{r}\right)=\min \left\{\frac{1}{r^{2}} \lambda_{1}\left(g_{1}\right), \lambda_{1}\left(g_{2}\right)\right\}=\frac{1}{r^{2}} \lambda_{1}\left(g_{1}\right)$. 
We have

$$
\operatorname{Ric}_{G_{r}}=\operatorname{Ric}_{g_{1}} \oplus \operatorname{Ric}_{g_{2}}=g_{1}+g_{2} \geq \frac{1}{r^{2}} G_{r},
$$

and $\Omega_{1}\left(G_{r}\right)=\Omega_{1}\left(g_{1}\right)$ by Proposition 2.14. Therefore, we get

$$
\frac{1}{r^{2}} \frac{1}{\Omega_{1}\left(g_{1}\right)}=\frac{1}{r^{2}} \lambda_{1}\left(g_{1}\right)=\lambda_{1}\left(G_{r}\right) .
$$

This gives an example mentioned above.

3.2. The proof of Theorem B. In this subsection, we prove Theorem B.

Theorem B . Let $(M, g)$ be a closed Riemannian manifold of dimension $n$. If $\Omega_{1}(g)=\Omega_{2}(g)=\frac{n-1}{n}$, then $(M, g)$ is isometric to the standard sphere of a certain radius.

The proof of this theorem depends on the following lemma. For a related work, see [9, Proposition 7.3].

Lemma 3.3. Let $(M, g)$ be a closed Riemannian manifold of dimension $n$. If there are linearly independent functions $u_{1}, u_{2} \in C^{\infty}(M) \cap H$ such that $\nabla^{2} u_{i}=$ $-\frac{\Delta u_{i}}{n} g(i=1,2)$, then $(M, g)$ is isometric to the standard sphere of a certain radius.

Before giving a proof of Lemma 3.3. we recall Tashiro's work [8]. First we enumerate the properties of the equation $\nabla^{2} u=-\frac{\Delta u}{n} g$.

Property A . If there exists a non-constant function $u \in C^{\infty}(M)$ such that $\nabla^{2} u=$ $-\frac{\Delta u}{n} g$, then $(M, g)$ and $u$ have the following properties:

(i) $u$ has just two critical points $\{p, q\}$ such that $u(p)<u(q)$.

(ii) There exist a constant $b>0$, a smooth function $\psi:[0, b] \rightarrow \mathbb{R}$ and $a$ diffeomorphism $\alpha: M \backslash\{p, q\} \rightarrow(0, b) \times S^{n-1}$ such that $u\left(\alpha^{-1}(t, x)\right)=$ $\psi(t)\left((t, x) \in(0, b) \times S^{n-1}\right)$ holds,

(iii) For any $t \in(0, b)$, we have $\psi^{\prime}(t)>0$. For any positive integer $k \in \mathbb{Z}_{>0}$, $\psi^{(2 k-1)}(0)=\psi^{(2 k-1)}(b)=0$ holds. Moreover, $\psi^{\prime \prime}(0)=-\psi^{\prime \prime}(b)>0$ holds,

(iv) Under the diffeomorphism $\alpha$, the metric $g$ on $M \backslash\{p, q\}$ is represented as $g(t, x)=d t^{2}+\frac{\psi^{\prime}(t)^{2}}{\psi^{\prime \prime}(0)^{2}} g_{n-1}(x)$, where $g_{n-1}$ denotes the standard metric on the sphere of radius 1 ,

(v) Define $P_{1}: M \backslash\{q\} \rightarrow B^{n}(b)$ and $P_{2}: M \backslash\{p\} \rightarrow B^{n}(b)$ by $P_{1}\left(\alpha^{-1}(t, x)\right)=$ $t x, P_{1}(p)=0$ and $P_{2}\left(\alpha^{-1}(t, x)\right)=(b-t) x, P_{2}(q)=0$ respectively, where $B^{n}(b)$ denotes the open ball of radius $b$ in $n$-dimensional Euclidean space with center 0 . Then, $P_{1}$ (resp. $P_{2}$ ) is the geodesic coordinate centered at $p$ (resp. q).

In particular, if there exists a non-constant function $u \in C^{\infty}(M)$ such that $\nabla^{2} u=-\frac{\Delta u}{n} g$, then $(M, g)$ is diffeomorphic to $S^{n}$ and has rotational symmetry. Let us explain why $(M, g)$ is conformal to the standard sphere. Fix $t_{0} \in(0, b)$ and put a $\operatorname{map} \theta:(0, b) \rightarrow(0, \pi)$ to be $\theta(t)=2 \arctan \exp \int_{t_{0}}^{t} \frac{\psi^{\prime \prime}(0)}{\psi^{\prime}(s)} d s$. By (iii), $\psi^{\prime}(s)=O(s)$ near $s=0$, and so $\lim _{t \rightarrow 0} \theta(t)=0$. Similarly, we have $\lim _{t \rightarrow b} \theta(t)=\pi$; therefore $\theta$ is a diffeomorphism. We have $\frac{d \theta}{d t}=\sin \theta \frac{\psi^{\prime \prime}(0)}{\psi^{\prime}(t)}$, and so $g(t, x)=\frac{1}{\sin ^{2} \theta} \frac{\psi^{\prime}(t)^{2}}{\psi^{\prime \prime}(0)^{2}}\left(d \theta^{2}+\right.$ $\left.\sin ^{2} \theta g_{n-1}\right)$. We know that $d \theta^{2}+\sin ^{2} \theta g_{n-1}$ is the standard metric on the sphere of radius 1. Moreover, $\frac{1}{\sin ^{2} \theta} \frac{\psi^{\prime}(t)^{2}}{\psi^{\prime \prime}(0)^{2}}$ can be extended to a smooth function on the whole $M$. This implies that $(M, g)$ is conformal to the standard sphere.

By the formulas for a warped product [1, Lemma 7.3] [4, Lemma 13], we have

Formulas . Let $X$ and $Y$ be vector fields on $M \backslash\{p, q\}$ that are orthogonal to $\frac{\partial}{\partial t}$.

(a) $\nabla_{X} Y(t, x)=-g(X, Y) \frac{\psi^{\prime \prime}(t)}{\psi^{\prime}(t)} \frac{\partial}{\partial t}+\nabla_{X}^{S^{n-1}} Y$, 
(b) $R^{M}\left(X, \frac{\partial}{\partial t}\right) \frac{\partial}{\partial t}=-\frac{\psi^{\prime \prime \prime}(t)}{\psi^{\prime}(t)} X$,

(c) $\operatorname{Ric}\left(\frac{\partial}{\partial t}, \frac{\partial}{\partial t}\right)(t)=-(n-1) \frac{\psi^{\prime \prime \prime}(t)}{\psi^{\prime}(t)}$,

where $R^{M}$ denotes the Riemann curvature tensor on $(M, g)$.

By (a), if $t_{1} \in(0, b)$ satisfies $\psi^{\prime \prime}\left(t_{1}\right)=0$, then the second fundamental form of the embedding $\left\{t_{1}\right\} \times S^{n-1} \subset M$ is equal to 0 . (b) immediately implies (c). By (c), $(M, g)$ has the following property:

Property B . For any normal geodesics $\gamma_{1}(t)$ and $\gamma_{2}(t)$ from $p \in M$, we have $\operatorname{Ric}\left(\dot{\gamma}_{1}(t), \dot{\gamma}_{1}(t)\right)=\operatorname{Ric}\left(\dot{\gamma}_{2}(t), \dot{\gamma}_{2}(t)\right)$.

Now, we are in position to prove Lemma 3.3 .

Proof of Lemma 3.3. We use the notations of Property A for $u_{1}$ : critical points $\{p, q\}$, a constant $b>0$, a diffeomorphism $\alpha: M \backslash\{p, q\}=(0, b) \times S^{n-1}$, and a map $\psi_{1}:[0, b] \rightarrow \mathbb{R}$ such that $u_{1}\left(\alpha^{-1}(t, x)\right)=\psi_{1}(t)\left((t, x) \in(0, b) \times S^{n-1}\right)$. Вy Property A (iii), we have $\psi_{1}^{\prime}(0)=\psi_{1}^{\prime}(b)=0$, and so there exists $t_{1} \in(0, b)$ such that $\psi_{1}^{\prime \prime}\left(t_{1}\right)=0$.

Let $p_{0}$ be one of critical points of $u_{2}$. Then, there exists a smooth map $\psi_{2}:[0, b] \rightarrow$ $\mathbb{R}$ such that $u_{2}(y)=\psi_{2}\left(d\left(p_{0}, y\right)\right)$. We extend $\psi_{2}:[-b, 2 b] \rightarrow \mathbb{R}$ by

$$
\psi_{2}(-t)=\psi_{2}(t), \psi_{2}(b+t)=\psi_{2}(b-t) \quad(t \in[0, b])
$$

Let us show $p \neq p_{0}$ by contradiction. Suppose that $p=p_{0}$. Then, by Property A (iv), we have $\frac{\psi_{1}^{\prime}(t)^{2}}{\psi_{1}^{\prime \prime}(0)^{2}}=\frac{\psi_{2}^{\prime}(t)^{2}}{\psi_{2}^{\prime \prime}(0)^{2}}$. Therefore, there exists a constant $C_{1} \in \mathbb{R}$ such that $\psi_{1}^{\prime}(t)=C_{1} \psi_{2}^{\prime}(t)$. Thus, there exists a constant $C_{2} \in \mathbb{R}$ such that $\psi_{1}(t)=C_{1} \psi_{2}(t)+$ $C_{2}$. Then, we have $C_{2}=u_{1}-C_{1} u_{2} \in H$, and so $C_{2}=0$. This is contradiction to the assumption of the linearly independence of $u_{1}$ and $u_{2}$. Therefore, we have $p \neq p_{0}$. Similarly, we have $q \neq p_{0}$.

We put $p_{0}=\left(t_{0}, x_{0}\right) \in M \backslash\{p, q\}$. We construct a non-constant function $u_{3}$ such that $\nabla^{2} u_{3}=-\frac{\Delta u_{3}}{n} g$ and $\alpha^{-1}\left(t_{1}, x_{0}\right)$ is one of critical points of $u_{3}$. If $t_{0}=t_{1}$, we put $u_{3}=u_{2}$. If $t_{0} \neq t_{1}$, we put $u_{3}=\psi_{2}^{\prime}\left(t_{1}-t_{0}\right) u_{1}-\psi^{\prime}\left(t_{1}\right) u_{2}$. Since $\psi_{1}^{\prime}\left(t_{1}\right) \neq 0$, we have $u_{3} \neq 0$. We have

$$
u_{3}\left(\alpha^{-1}\left(t, x_{0}\right)\right)=\psi_{2}^{\prime}\left(t_{1}-t_{0}\right) \psi_{1}(t)-\psi^{\prime}\left(t_{1}\right) \psi_{2}\left(t-t_{0}\right)
$$

and so $\frac{\partial}{\partial t} u_{3}\left(\alpha^{-1}\left(t_{1}, x_{0}\right)\right)=0$. For any tangent vector $X \in T_{\alpha^{-1}\left(t_{1}, x_{0}\right)} M$ that is orthogonal to $\left(\frac{\partial}{\partial t}\right)_{\left(t_{1}, x_{0}\right)}$, we have $X u_{1}\left(\alpha^{-1}\left(t_{1}, x_{0}\right)\right)=X u_{2}\left(\alpha^{-1}\left(t_{1}, x_{0}\right)\right)=0$, and so $X u_{3}\left(\alpha^{-1}\left(t_{1}, x_{0}\right)\right)=0$. Therefore, $\alpha^{-1}\left(t_{1}, x_{0}\right)$ is one of critical points of $u_{3}$. In both cases, $u_{3}$ has desired property.

Since $\psi_{1}^{\prime \prime}\left(t_{1}\right)=0$, the second fundamental form of the embedding $\left\{t_{1}\right\} \times S^{n-1} \subset$ $M$ is equal to 0 . Therefore, for any normal geodesic $\gamma(s)$ in $S^{n-1}(1)$ from $x_{0} \in S^{n-1}$, $\tilde{\gamma}(s)=\alpha^{-1}\left(t_{1}, \gamma\left(\frac{\psi_{1}^{\prime \prime}(0)}{\psi_{1}^{\prime}\left(t_{1}\right)} s\right)\right) \in M$ is a normal geodesic in $M$ from $\alpha^{-1}\left(t_{1}, x_{0}\right)$. By the symmetry, there exists a constant $C_{3} \in \mathbb{R}$ such that

$$
\operatorname{Ric}(\dot{\tilde{\gamma}}(s), \dot{\tilde{\gamma}}(s))=C_{3}
$$

holds. 


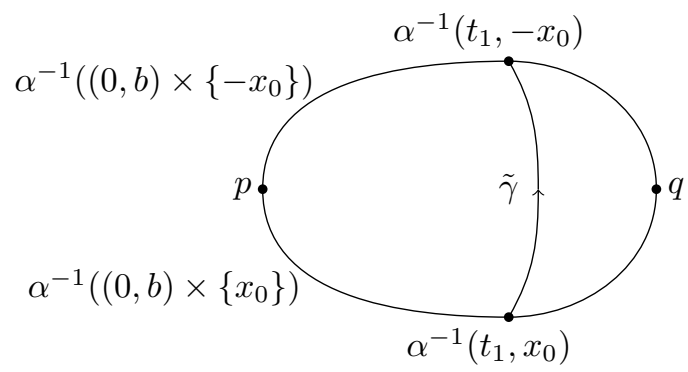

By Property B for $u_{3}$, we have

$$
-(n-1) \frac{\psi_{1}^{\prime \prime \prime}}{\psi_{1}^{\prime}}=\operatorname{Ric}\left(\frac{\partial}{\partial t}, \frac{\partial}{\partial t}\right)=\operatorname{Ric}(\dot{\tilde{\gamma}}(s), \dot{\tilde{\gamma}}(s))=C_{3} .
$$

By (11) and Property A (iii), there exists a constant $C_{4}$ such that $\psi_{1}^{\prime}(t)=$ $C_{4} \sin \left(\frac{\pi}{b} t\right)$. Therefore, the metric $g$ on $M$ is represented as

$$
g(t, x)=d t^{2}+\frac{b^{2}}{\pi^{2}} \sin ^{2}\left(\frac{\pi}{b} t\right) g_{n-1} .
$$

Putting $\theta=\frac{\pi}{b} t$, we get $g=\frac{b^{2}}{\pi^{2}}\left(d \theta^{2}+\sin ^{2} \theta g_{n-1}\right)$. This is the standard metric on the sphere of radius $\frac{b}{\pi}$.

Proof of Theorem B. Lemma 3.3 immediately implies the theorem.

3.3. An estimate in the presence of a parallel $p$-form. The goal of this subsection is to prove the following theorem.

Theorem 3.4. Let $(M, g)$ be a closed Riemannian manifold of dimension $n=2 p$ $(p \geq 2)$. If there exists a nontrivial parallel $p$-form $\omega$ on $M$, then we have $\Omega_{1}(g) \leq$ $\frac{p-1}{p}$.

The proof of this theorem depends on the method used in 3 . Before giving the proof, we recall some definitions and properties about $p$-form. Let $\omega$ be a $p$ form on $M$ and $\left\{e_{i}\right\}_{1 \leq i \leq n}$ a local orthonormal frame. We write $\omega_{i_{1} \cdots i_{p}}$ instead of $\omega\left(e_{i_{1}}, \cdots, e_{i_{p}}\right)$. We define the inner product of $p$-forms $\omega$ and $\theta$ by

$$
(\omega, \theta)=\frac{1}{p !} \sum_{1 \leq i_{1}<\ldots<i_{p} \leq n} \omega_{i_{1} \cdots i_{p}} \theta_{i_{1} \cdots i_{p}} .
$$

The inner product of a $p$-form $\omega$ with the vector field $X$ is the $(p-1)$-form $\iota(X) \omega$ defined by

$$
(\iota(X) \omega)\left(Y_{1}, \cdots, Y_{p-1}\right)=\omega\left(X, Y_{1}, \cdots, Y_{p-1}\right),
$$

for any vector field $Y_{1}, \cdots, Y_{p-1}$. For any vector field $X$, we define a 1 -form $X^{*}$ by

$$
X^{*}(Y)=g(X, Y)
$$

for any vector field $Y$.

Suppose that $M$ is orientable. Then, for any vector field $X, p$-form $\omega$ and $(p-1)$ form $\eta$, we have

$$
\begin{array}{r}
\left(*\left(\omega \wedge X^{*}\right), \eta\right) d \mu_{g}=\omega \wedge X^{*} \wedge \eta \\
(\iota(X) * \omega, \eta) d \mu_{g}=\left(* \omega, X^{*} \wedge \eta\right) d \mu_{g}=\omega \wedge X^{*} \wedge \eta,
\end{array}
$$

where $*$ denotes the Hodge star operator and $d \mu_{g}$ denotes the volume form on $(M, g)$. Thus, we have $*\left(\omega \wedge X^{*}\right)=\iota(X) * \omega$. Therefore, for any vector field $X, Y$ 
and a $p$-form $\omega$, we have

$$
\begin{aligned}
& \iota(X) \omega, \iota(Y) \omega) \\
= & \left(\omega, X^{*} \wedge \iota(Y) \omega\right) \\
= & \left(\omega,-\iota(Y)\left(X^{*} \wedge \omega\right)+(X, Y) \omega\right)=-\left(Y^{*} \wedge \omega, X^{*} \wedge \omega\right)+(X, Y)(\omega, \omega) \\
= & -(\iota(X) * \omega, \iota(Y) * \omega)+(X, Y)(\omega, \omega)
\end{aligned}
$$

For any function $u \in C^{\infty}(M)$ and $p$-form $\omega$, we define $p$-tensor $D_{\omega} u$ by

$$
\begin{aligned}
D_{\omega} u= & \frac{1}{(p-2) !} \sum_{i, i_{1}, \cdots, i_{p-2}}\left(\iota\left(e_{i}\right) \iota\left(e_{i_{1}}\right) \cdots \iota\left(e_{i_{p-2}}\right) \omega \otimes \nabla_{e_{i}} d u\right. \\
& \left.+\nabla_{e_{i}} d u \otimes \iota\left(e_{i}\right) \iota\left(e_{i_{1}}\right) \cdots \iota\left(e_{i_{p-2}}\right) \omega\right) \otimes\left(e^{i_{1}} \wedge \cdots \wedge e^{i_{p-2}}\right) .
\end{aligned}
$$

Put

$$
\begin{aligned}
\left(D_{\omega} u\right)_{j k i_{1}, \cdots, i_{p-2}} & =D_{\omega} u\left(e_{j}, e_{k}, e_{i_{1}}, \cdots, e_{i_{p-2}}\right) \\
\left|D_{\omega} u\right|^{2} & =\frac{1}{(p-2) !} \sum_{j k i_{1} \cdots i_{p-2}}\left(D_{\omega} u\right)_{j k i_{1}, \cdots, i_{p-2}}^{2}
\end{aligned}
$$

If $\omega$ is a parallel $p$-form, then by [3, Proposition 3.1], we have

$$
\begin{aligned}
& \frac{1}{p} \int_{M}\left|D_{\omega} u\right|^{2} d \mu_{g} \\
= & \frac{p-1}{p} \int_{M}(\iota(\nabla u) \omega, \iota(\nabla \Delta u) \omega) d \mu_{g}-\int_{M}(\iota(\nabla u) \omega, \iota(\operatorname{Ric}(\nabla u, \cdot)) \omega) d \mu_{g} .
\end{aligned}
$$

Proof of Theorem 3.4. If $M$ is not orientable, we take an orientable double covering $P: \widetilde{M} \rightarrow M$ and consider the metric $\tilde{g}=P^{*} g$ on $\widetilde{M}$. Suppose $\Omega_{1}(g)>0$ and take a function $v \in H_{(M, g)}$ such that $\Delta^{2} v=\frac{1}{\Omega_{1}(g)} \nabla^{*}\left(\operatorname{Ric}_{g}(\nabla v)\right)$. Then, we have $\Delta^{2}(v \circ P)=\frac{1}{\Omega_{1}(g)} \nabla^{*}\left(\operatorname{Ric}_{\tilde{g}}(\nabla(v \circ P))\right)$. Therefore, $\Omega_{1}(g) \leq \Omega_{1}(\tilde{g})$ holds. If $\Omega_{1}(g)=0$, we obviously have $\Omega_{1}(g) \leq \Omega_{1}(\tilde{g})$. Thus, it is suffice to consider the case when $M$ is orientable.

Suppose that $M$ is orientable. Take a function $u \in C^{\infty}(M)$. Since $\omega$ and $* \omega$ is parallel, by (13) we have

$$
\begin{aligned}
0 \leq & \frac{1}{p} \int_{M}\left|D_{\omega} u\right|^{2} d \mu_{g}+\frac{1}{p} \int_{M}\left|D_{* \omega} u\right|^{2} d \mu_{g} \\
= & \frac{p-1}{p} \int_{M}(\iota(\nabla u) \omega, \iota(\nabla \Delta u) \omega) d \mu_{g}+\frac{p-1}{p} \int_{M}(\iota(\nabla u) * \omega, \iota(\nabla \Delta u) * \omega) d \mu_{g} \\
& -\int_{M}(\iota(\nabla u) \omega, \iota(\operatorname{Ric}(\nabla u, \cdot)) \omega) d \mu_{g}-\int_{M}(\iota(\nabla u) * \omega, \iota(\operatorname{Ric}(\nabla u, \cdot)) * \omega) d \mu_{g} .
\end{aligned}
$$

Note that we assumed $n=2 p$, and so $* \omega$ is a $p$-form.

By (12) and (14), we obtain

$$
\begin{aligned}
0 & \leq \frac{p-1}{p} \int_{M}(\nabla u, \nabla \Delta u) d \mu_{g}|\omega|^{2}-\int_{M} \operatorname{Ric}(\nabla u, \nabla u) d \mu_{g}|\omega|^{2} \\
& =\frac{p-1}{p} \int_{M}(\Delta u, \Delta u) d \mu_{g}|\omega|^{2}-\int_{M} \operatorname{Ric}(\nabla u, \nabla u) d \mu_{g}|\omega|^{2}
\end{aligned}
$$

Consequently, for any $v \in H \backslash\{0\}$, we have $\Lambda_{\text {Ric }}(v) \leq \frac{p-1}{p}$. Therefore, $\Omega_{1}(g) \leq$ $\frac{p-1}{p}$ holds. 
3.4. Riemannian manifolds whose value of $\Omega_{1}$ is close to $\frac{n-1}{n}$. In this subsection, we give an example of a Riemannian manifold that is far from $S^{n}$ but whose value of $\Omega_{1}$ is close to $\frac{n-1}{n}$.

Let $(M, g)$ be a $n$-dimensional closed Riemannian manifold with rotational symmetry, i.e., there exists a non-constant function $u \in C^{\infty}(M)$ such that $\nabla^{2} u=$ $-\frac{\Delta u}{n} g$, and so Property A holds. We use the notations of Property A: critical points $\{p, q\}$, a constant $b>0$, a diffeomorphism $\alpha: M \backslash\{p, q\}=(0, b) \times S^{n-1}$, and a map $\psi:[0, b] \rightarrow \mathbb{R}$ such that $u\left(\alpha^{-1}(t, x)\right)=\psi(t)\left((t, x) \in(0, b) \times S^{n-1}\right)$. We may assume $u(p)=0$. There exists a positive constant $C_{1}>0$ such that

$$
\left|\psi^{\prime}(t)\right| \leq C_{1}, \quad\left|\psi^{\prime \prime}(t)\right| \leq C_{1}, \quad\left|\psi^{(4)}(t)\right| \leq C_{1} .
$$

Since $\psi(0)=0, \psi^{\prime}(0)=0$ and $\psi^{\prime \prime \prime}(0)=0$, we have

$$
|\psi(t)| \leq C_{1} t^{2}, \quad\left|\psi^{\prime}(t)\right| \leq C_{1} t, \quad\left|\psi^{\prime \prime \prime}(t)\right| \leq C_{1} t .
$$

For any $R>0$, we define

$$
B^{M}(q, R)=\left\{z \in M: d^{M}(q, z)<R\right\} .
$$

Take a $n$-dimensional closed Riemannian manifold $\left(N, g^{\prime}\right)$ and a point $y_{0} \in N$. Let $\left(U ; x^{1}, \cdots, x^{n}\right)$ be a local coordinate centered at $y_{0}$. Take a positive real number $L$ such that

$$
\overline{B^{n}(2 L)} \subset U
$$

where $B^{n}(2 L)$ denotes the open ball in $n$-dimensional Euclidean space of radius $2 L$.

For any $l \in(0, L)$, define $\theta_{l}: N \rightarrow \mathbb{R}$ by

$$
\theta_{l}(y)=\left\{\begin{array}{cl}
0 & \left(y \in \overline{B^{n}(l)}\right), \\
0<\theta_{l}(y) \leq 1 & \left(y \in B^{n}(2 l) \backslash \overline{B^{n}(l)}\right) \\
1 & \left(y \in N \backslash B^{n}(2 l)\right) .
\end{array}\right.
$$

Take a smooth function $\phi: \mathbb{R} \rightarrow \mathbb{R}$ such that

$$
\phi(t)=\left\{\begin{array}{cl}
0 & \left(t<\frac{3}{2}\right), \\
0 \leq \phi(t) \leq 1 & \left(\frac{3}{2} \leq t \leq 2\right), \\
1 & (2<t) .
\end{array}\right.
$$

There exist a positive constant $C_{2}>0$ such that $\sup _{t \in \mathbb{R}}\left|\phi^{\prime}(t)\right| \leq C_{2}$.

For any $l \in(0, L)$ and small $\epsilon>0$, there exists a diffeomorphism

$$
\psi_{(l, \epsilon)}: B^{M}(q, b-\epsilon) \rightarrow B^{n}(2 l) \subset N
$$

such that $\psi_{(l, \epsilon)}\left(B^{M}(q, b-2 \epsilon)\right)=B^{n}(l)$. Define $\zeta_{\epsilon}, \eta_{\epsilon}: M \rightarrow \mathbb{R}$ by

$$
\begin{aligned}
& \zeta_{\epsilon}(z)=\phi\left(\frac{d^{M}(z, p)}{\epsilon}\right), \\
& \eta_{\epsilon}(z)=\phi\left(\frac{d^{M}(z, p)}{2 \epsilon}\right) .
\end{aligned}
$$

For any $r>0, l \in(0, L)$ and small $\epsilon>0$, we define a metric on $N$ by

$$
g_{(r, l, \epsilon)}=\left(\psi_{(l, \epsilon)}^{-1}\right)^{*}\left(r \zeta_{\epsilon} g\right)+\theta_{l} g^{\prime} .
$$




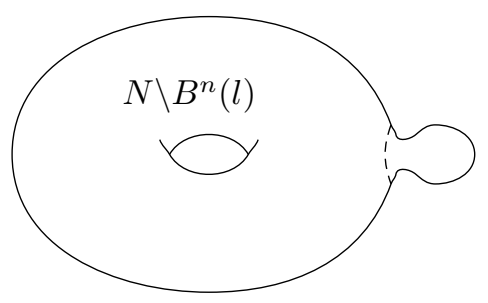

We put $u_{(l, \epsilon)}=\left(\psi_{(l, \epsilon)}^{-1}\right)^{*}\left(\eta_{\epsilon} u\right) \in C^{\infty}(N)$. Then, we have

$$
\Lambda_{\operatorname{Ric}_{g_{(r, l, \epsilon)}}}\left(u_{(l, \epsilon)}\right)=\frac{\int_{M} \operatorname{Ric}_{g}\left(\nabla^{g}\left(\eta_{\epsilon} u\right), \nabla^{g}\left(\eta_{\epsilon} u\right)\right) d \mu_{g}}{\int_{M}\left(\Delta^{g}\left(\eta_{\epsilon} u\right)\right)^{2} d \mu_{g}} .
$$

By the formulas of a warped product and (15), for some positive constant $C_{3}>0$, we have

$$
\begin{aligned}
& \left|\int_{B^{M}(p, 4 \epsilon)} \operatorname{Ric}_{g}\left(\nabla^{g}\left(\eta_{\epsilon} u\right), \nabla^{g}\left(\eta_{\epsilon} u\right)\right) d \mu_{g}\right| \\
= & (n-1) \operatorname{Vol}\left(S^{n-1}(1)\right) \\
& \times\left|\int_{3 \epsilon}^{4 \epsilon} \frac{\psi^{\prime \prime \prime}(t)}{\psi^{\prime}(t)}\left(\frac{1}{2 \epsilon} \phi^{\prime}\left(\frac{t}{2 \epsilon}\right) \psi(t)+\phi\left(\frac{t}{2 \epsilon}\right) \psi^{\prime}(t)\right)^{2}\left(\frac{\psi^{\prime}(t)}{\psi^{\prime \prime}(0)}\right)^{n-1} d t\right| \\
\leq & C_{3} \epsilon^{n+2} \rightarrow 0 \quad(\epsilon \rightarrow 0) .
\end{aligned}
$$

Similarly, for some positive constant $C_{4}>0$, we have

$$
\left|\int_{B^{M}(p, 4 \epsilon)}\left(\Delta^{g}\left(\eta_{\epsilon} u\right)\right)^{2} d \mu_{g}\right| \leq C_{4} \epsilon^{n} \rightarrow 0 \quad(\epsilon \rightarrow 0)
$$

Note that $\eta_{\epsilon} u=u$ on $M \backslash B^{M}(p, 4 \epsilon)$. By (16), (17) and (18), we have

$$
\begin{aligned}
& \frac{n-1}{n} \\
\geq & \Omega_{1}\left(g_{(r, l, \epsilon)}\right) \\
\geq & \frac{\int_{M} \operatorname{Ric}_{g}\left(\nabla^{g}\left(\eta_{\epsilon} u\right), \nabla^{g}\left(\eta_{\epsilon} u\right)\right) d \mu_{g}}{\int_{M}\left(\Delta^{g}\left(\eta_{\epsilon} u\right)\right)^{2} d \mu_{g}} \\
\rightarrow & \frac{\int_{M} \operatorname{Ric}_{g}\left(\nabla^{g} u, \nabla^{g} u\right) d \mu_{g}}{\int_{M}\left(\Delta^{g} u\right)^{2} d \mu_{g}}=\frac{n-1}{n},
\end{aligned}
$$

as $\epsilon \rightarrow 0$. Therefore, we have the following theorem.

Theorem 3.5. The above notations are preserved. For any sequence $\left\{\left(r_{i}, l_{i}, \epsilon_{i}\right)\right\}_{i \in \mathbb{N}}$ such that $\epsilon_{i} \rightarrow 0$ as $i \rightarrow \infty$, we have $\lim _{i \rightarrow \infty} \Omega_{1}\left(g_{\left(r_{i}, l_{i}, \epsilon_{i}\right)}\right)=\frac{n-1}{n}$.

Remark 3.2. For any sequence $\left\{\left(r_{i}, l_{i}, \epsilon_{i}\right)\right\}_{i \in \mathbb{N}}$ such that $\left(r_{i}, l_{i}, \epsilon_{i}\right) \rightarrow(0,0,0)$ as $i \rightarrow$ $\infty$, we have $\left(N, g_{\left(r_{i}, l_{i}, \epsilon_{i}\right)}\right) \rightarrow\left(N, g^{\prime}\right)$ in the Gromov-Hausdorff topology. However, $\lim _{i \rightarrow \infty} \Omega_{1}\left(g_{\left(r_{i}, l_{i}, \epsilon_{i}\right)}\right)=\frac{n-1}{n}$ is not necessarily $\Omega_{1}\left(g^{\prime}\right)$.

Corollary 3.6. For any closed manifold $N$ of dimension $n$, we have

$$
\sup _{g \in \operatorname{Met}(N)} \Omega_{1}(g)=\frac{n-1}{n},
$$

where $\operatorname{Met}(N)$ denotes the set of all Riemannian metrics on $N$. 


\section{Computation And examples}

4.1. The product of Einstein manifolds. In general, it is difficult to calculate $\Omega_{k}$ for a product Riemannian manifold. However, we have the following theorem.

Theorem 4.1. Let $\left(M_{i}, g_{i}\right)$ be closed Einstein manifolds: $\operatorname{Ric}_{g_{i}}=a_{i} g_{i}$. Consider the product metric $g=g_{1}+g_{2}$ on $M=M_{1} \times M_{2}$. Assume that $a_{1}>0$. Then, we have

$$
\left\{\Omega_{l}(g): l \in \mathbb{Z}_{>0}\right\}=\left\{\frac{\left(a_{1} \lambda_{i}+a_{2} \lambda_{k}^{\prime}\right)}{\left(\lambda_{i}+\lambda_{k}^{\prime}\right)^{2}}:(i, k) \neq(0,0) \text { and } \frac{\left(a_{1} \lambda_{i}+a_{2} \lambda_{k}^{\prime}\right)}{\left(\lambda_{i}+\lambda_{k}^{\prime}\right)^{2}}>0\right\},
$$

where $\left\{\lambda_{i}\right\}$ (resp. $\left\{\lambda_{k}^{\prime}\right\}$ ) are the eigenvalues of the Laplacian of $\left(M_{1}, g_{1}\right)$ (resp. $\left.\left(M_{2}, g_{2}\right)\right)$.

Proof. Let $\left\{\psi_{i}\right\}$ (resp. $\left.\left\{\psi_{k}^{\prime}\right\}\right)$ be the eigenfunctions of the Laplacian of $\left(M_{1}, g_{1}\right)$ (resp. $\left.\left(M_{2}, g_{2}\right)\right)$. Then, $\left\{\frac{1}{\lambda_{i}+\lambda_{k}^{\prime}} \psi_{i} \psi_{k}^{\prime}\right\}_{(i, k) \neq(0,0)}$ forms a complete orthonormal system of $H_{(M, g)}$. Moreover, we have

$$
\nabla^{*}\left(\operatorname{Ric}_{g}\left(\nabla\left(\psi_{i} \psi_{k}^{\prime}\right), \cdot\right)\right)=\left(a_{1} \lambda_{i}+a_{2} \lambda_{k}^{\prime}\right) \psi_{i} \psi_{k}^{\prime}=\frac{\left(a_{1} \lambda_{i}+a_{2} \lambda_{k}^{\prime}\right)}{\left(\lambda_{i}+\lambda_{k}^{\prime}\right)^{2}} \Delta^{2}\left(\psi_{i} \psi_{k}^{\prime}\right) .
$$

Therefore, by Corollary 2.12, we get

$$
\left\{\Omega_{l}(g): l \in \mathbb{Z}_{>0}\right\}=\left\{\frac{\left(a_{1} \lambda_{i}+a_{2} \lambda_{k}^{\prime}\right)}{\left(\lambda_{i}+\lambda_{k}^{\prime}\right)^{2}}:(i, k) \neq(0,0) \text { and } \frac{\left(a_{1} \lambda_{i}+a_{2} \lambda_{k}^{\prime}\right)}{\left(\lambda_{i}+\lambda_{k}^{\prime}\right)^{2}}>0\right\} .
$$

Example 4.1. By using Theorem 4.1. we calculate the value of $\Omega_{1}$ of the unitary group $U(n)$ with bi-invariant metrics.

We know that $U(n)=(\mathbb{T} \times S U(n)) / Z$, where $\mathbb{T}=\{t \in \mathbb{C}:|t|=1\}=S^{1}$ and $Z=\left\{\left(e^{-2 \pi \sqrt{-1} k / n}, e^{2 \pi \sqrt{-1} k / n}\right) \in \mathbb{T} \times S U(n): k=0,1, \ldots, n-1\right\}$. Let $g_{0}$ be the bi-invariant metric on $S U(n)$ defined by

$$
g_{0}(X, Y)=-\operatorname{tr}(X Y) \quad X, Y \in \mathfrak{s u}(n) .
$$

Then, we have

$$
\operatorname{Ric}_{g_{0}}=\frac{1}{2} n g_{0} .
$$

Let $\widetilde{G}_{r}$ be a bi-invariant metric on $\mathbb{T} \times S U(n)$ defined by

$$
\widetilde{G}_{r}=g_{1}(r)+g_{0}
$$

for a positive real number $r \in \mathbb{R}_{>0}$, where $g_{1}(r)$ is the standard metric on $S^{1}$ of radius $r$. Let $G_{r}$ be a bi-invariant metric on $U(n)$ induced by $\widetilde{G}_{r}$. Note that $G_{\sqrt{n}}$ coincides with the metric on $U(n)$ defined by

$$
-\operatorname{tr}(X Y) \quad X, Y \in \mathfrak{u}(n) \text {. }
$$

We show that $\Omega_{1}\left(G_{r}\right)=\max \left\{\frac{\frac{1}{2} n^{2}\left(n^{2}-1\right)}{\left(n^{2}+\frac{n}{r^{2}}-1\right)^{2}}, \frac{1}{4}\right\}$.

To do this, we recall the fact about the eigenvalues of $\left(S U(n), g_{0}\right)$. We can compute the spectrum of compact Lie groups by a representation theoretic way, see e.g. [7, Lemma 1.1]. We only describe some results about $\left(S U(n), g_{0}\right)$. Let $\left\{\lambda_{k}\left(g_{0}\right)\right\}$ be the eigenvalues of $\left(S U(n), g_{0}\right)$. Then, $\lambda_{1}\left(g_{0}\right)=n-\frac{1}{n}$ holds, and the first eigenfunction is not $Z$-invariant on $\mathbb{T} \times S U(n)$. We can choose the first eigenfunction $\psi_{1}$ on $\left(S U(n), g_{0}\right)$ such that $\widetilde{\psi}_{1}(t, x)=t \psi_{1}(x)((t, x) \in \mathbb{T} \times S U(n))$ is $Z$-invariant. On the other hand, we have

$2 n=\min \left\{\lambda_{k}\left(g_{0}\right): k \in \mathbb{Z}_{>0}\right.$ and the eigenfunction is $Z$-invariant on $\left.\mathbb{T} \times S U(n)\right\}$.

Let $\widetilde{\psi_{2}}$ be such an eigenfunction. 
By Theorem 4.1, the associated functions (see Definition 1) on $\mathbb{T} \times S U(n)$ are of the form

$$
t^{k} \psi_{i}(x) \quad(t, x) \in \mathbb{T} \times S U(n)
$$

for some integer $k \in \mathbb{Z}$ and some eigenfunction $\psi_{i}$ on $S U(n)$ such that $\Delta \psi_{i}=$ $\lambda_{i}\left(g_{0}\right) \psi_{i}$. Then,

$$
\nabla^{*}\left(\operatorname{Ric}_{\widetilde{G}_{r}}\left(\nabla\left(t^{k} \psi_{i}\right), \cdot\right)\right)=\frac{1}{2} n \lambda_{i} t^{k} \psi_{i}=\frac{\frac{1}{2} n \lambda_{i}}{\left(\frac{1}{r^{2}} k^{2}+\lambda_{i}\right)^{2}} \Delta^{2}\left(t^{k} \psi_{i}\right) .
$$

Moreover, we have

$\Omega_{1}\left(G_{r}\right)=\max \left\{\Omega_{k}\left(\widetilde{G}_{r}\right): k \in \mathbb{Z}_{>0}\right.$ and the associated function $v_{k}$ is $Z$-invariant $\}$.

Thus, the candidates of $\Omega_{1}\left(G_{r}\right)$ are $\Lambda_{\operatorname{Ric}_{\widetilde{G}_{r}}}\left(\widetilde{\psi}_{1}\right)$ and $\Lambda_{\operatorname{Ric}_{\widetilde{G}_{r}}}\left(\widetilde{\psi}_{2}\right)$. We have

$$
\begin{aligned}
& \nabla^{*}\left(\operatorname{Ric}_{\widetilde{G}_{r}}\left(\nabla \widetilde{\psi}_{1}, \cdot\right)\right)=\frac{\frac{1}{2} n\left(n-\frac{1}{n}\right)}{\left(\frac{1}{r^{2}}+n-\frac{1}{n}\right)^{2}} \Delta^{2} \widetilde{\psi}_{1}=\frac{\frac{1}{2} n^{2}\left(n^{2}-1\right)}{\left(n^{2}+\frac{n}{r^{2}}-1\right)^{2}} \Delta^{2} \widetilde{\psi}_{1}, \\
& \nabla^{*}\left(\operatorname{Ric}_{\widetilde{G}_{r}}\left(\nabla \widetilde{\psi}_{2}, \cdot\right)\right)=\frac{1}{4} \Delta^{2} \widetilde{\psi}_{2} .
\end{aligned}
$$

Therefore, we get

$$
\Omega_{1}\left(G_{r}\right)=\max \left\{\frac{\frac{1}{2} n^{2}\left(n^{2}-1\right)}{\left(n^{2}+\frac{n}{r^{2}}-1\right)^{2}}, \frac{1}{4}\right\} .
$$

4.2. The case of Heisenberg manifolds. In this subsection, we consider the value of $\Omega_{1}$ of the Heisenberg manifolds. We refer to [2]. Let $n \in \mathbb{Z}_{>0}$. For $x, y \in \mathbb{R}^{n}$ and $t \in \mathbb{R}$, let

$$
\gamma(x, y, t)=\left[\begin{array}{ccc}
1 & x & t \\
0 & I_{n} & { }^{t} y \\
0 & 0 & 1
\end{array}\right], \quad X(x, y, t)=\left[\begin{array}{ccc}
0 & x & t \\
0 & 0 & { }^{t} y \\
0 & 0 & 0
\end{array}\right]
$$

where we consider $x, y$ as law vectors. The Heisenberg group $H_{n}$ is the $(2 n+1)$ dimensional Lie group defined by

$$
H_{n}=\left\{\gamma(x, y, t) \in G L(n+2, \mathbb{R}): x, y \in \mathbb{R}^{n} \text { and } t \in \mathbb{R}\right\},
$$

with Lie algebra

$$
\mathfrak{h}_{n}=\left\{X(x, y, t) \in M(n+2, \mathbb{R}): x, y \in \mathbb{R}^{n} \text { and } t \in \mathbb{R}\right\} .
$$

Let $\Gamma$ be a discrete subgroup of $H_{n}$ such that $M=\Gamma \backslash H_{n}$ is compact (such a subgroup is called a uniform discrete subgroup). We call such a manifold $M$ a Heisenberg manifold. Let $\mathrm{L}(M)$ be the set of all left invariant metrics on $M$. Then, we have the following proposition.

Proposition 4.2. For any Heisenberg manifolds $M=\Gamma \backslash H_{n}$, we have

$$
\sup _{g \in \mathrm{L}(M)} \Omega_{1}(g)= \begin{cases}\frac{1}{16} \frac{\sqrt{17}-3}{(\sqrt{17}-1)(\sqrt{17}+3)^{2}} & n=1 \\ \frac{1}{32} & n \geq 2 .\end{cases}
$$

Remark 4.1. We have

$$
\begin{aligned}
& \inf \left\{\lambda_{1}(g): g \in \mathrm{L}(M) \text { and } \operatorname{vol}(M, g)=1\right\}=0, \\
& \sup \left\{\lambda_{1}(g): g \in \mathrm{L}(M) \text { and } \operatorname{vol}(M, g)=1\right\}=\infty
\end{aligned}
$$

Before giving a proof of the proposition, we recall the basic results described in [2] and some other facts. 
Notation 3. We put

$$
X_{i}=X\left(e_{i}, 0,0\right), \quad Y_{i}=X\left(0, e_{i}, 0\right), \quad Z=X(0,0,1)
$$

for $1 \leq i \leq n$, where $\left\{e_{i}\right\}$ denotes the standard basis of $\mathbb{R}^{n}$. We put

$$
\begin{aligned}
\mathfrak{z}_{n} & =\{t Z: t \in \mathbb{R}\}, \\
\mathfrak{z}_{n}^{\perp} & =\operatorname{Span}_{\mathbb{R}}\left\{X_{1}, \ldots, X_{n}, Y_{1}, \ldots, Y_{n}\right\} .
\end{aligned}
$$

Note that $\mathfrak{z}_{n}$ is the center of $\mathfrak{h}_{n}$, and

$$
\left[X_{i}, X_{j}\right]=\left[Y_{i}, Y_{j}\right]=0, \quad\left[X_{i}, Y_{j}\right]=\delta_{i j} Z
$$

for $1 \leq i, j \leq n$.

Notation 4. We put

$$
\mathbb{Z}_{\text {div }}^{n}=\left\{r=\left(r_{1}, \ldots, r_{n}\right) \in \mathbb{Z}_{>0}^{n}: r_{j} \text { divides } r_{j+1} \text { for } 1 \leq j \leq n-1\right\} .
$$

For $r \in \mathbb{Z}_{\text {div }}^{n}$, we put

$$
\begin{aligned}
r \mathbb{Z}^{n} & =\left\{x=\left(x_{1}, \ldots, x_{n}\right) \in \mathbb{Z}^{n}: x_{i} \in r_{i} \mathbb{Z} \text { for } 1 \leq i \leq n\right\}, \\
\Gamma_{r} & =\left\{\gamma(x, y, t) \in H_{n}: x \in r \mathbb{Z}^{n}, y \in \mathbb{Z}^{n}, t \in \mathbb{Z}\right\}, \\
\mathcal{A}_{r} & =\left\{\tau \in \mathfrak{h}_{n}^{*}: \tau(Z)=0 \text { and } \tau\left(\log \Gamma_{r}\right) \subset \mathbb{Z}\right\} .
\end{aligned}
$$

By [2, Lemma 3.5], for any uniform discrete subgroup $\Gamma$ of $H_{n}$, there exist an automorphism $\psi: H_{n} \rightarrow H_{n}$ and $r \in \mathbb{Z}_{\text {div }}^{n}$ such that

$$
\psi(\Gamma)=\Gamma_{r}
$$

holds. Therefore, $\Gamma \backslash H_{n}$ is identified with $\Gamma_{r} \backslash H_{n}$, and so it is suffice to consider the case when $\Gamma=\Gamma_{r}$.

By the [2, Remark 2.6], for any metric $g$ on $\mathfrak{h}_{n}=\mathfrak{z}_{n}^{\perp} \oplus \mathfrak{z}_{n}$, there exists an inner automorphism $\varphi: H_{n} \rightarrow H_{n}$ such that $\varphi^{*} g$ is of the form

$$
\varphi^{*} g=\left[\begin{array}{cc}
h & 0 \\
0 & g_{n+1}
\end{array}\right]
$$

with $h$ a metric on $\mathfrak{z}_{n}^{\perp}$ and $g_{2 n+1}>0$. By [2, Proposition 2.2], $\left(\Gamma_{r} \backslash H_{n}, g\right)$ is isometric to $\left(\Gamma_{r} \backslash H_{n}, \varphi^{*} g\right)$. Thus, it is suffice to consider the case when $g$ is of the form

$$
g=\left[\begin{array}{cc}
h & 0 \\
0 & g_{n+1}
\end{array}\right] \text {. }
$$

We next explain the fact about the irreducible unitary representations of $H_{n}$.

Notation 5. We consider the following irreducible unitary representations of $H_{n}$.

(a) For $\tau \in \mathfrak{h}_{n}^{*}$ with $\tau(Z)=0$, we put $f_{\tau}: H_{n} \rightarrow U(1)$ by $f_{\tau}(\exp X)=$ $\exp (2 \pi \sqrt{-1} \tau(X))$ for all $X \in \mathfrak{h}_{n}$. Then, $f_{\tau}$ defines a 1-dimensional unitary representation of $H_{n}$.

(b) For $c \in \mathbb{R} \backslash\{0\}$, we define a representation $\pi_{c}$ on $L^{2}\left(\mathbb{R}^{n}\right)$ by

$$
\left(\pi_{c}(\gamma(x, y, t)) f\right)(u)=\exp (2 \pi \sqrt{-1} c(t+u \cdot y)) f(x+u)
$$

for all $f \in L^{2}\left(\mathbb{R}^{n}\right)$ and $\gamma(x, y, t) \in H_{n}$.

By [2, Lemma 3.7],

$$
\left\{f_{\tau}: \tau \in \mathfrak{h}_{n}^{*} \text { and } \tau(Z)=0\right\} \cup\left\{\pi_{c}: c \in \mathbb{R} \backslash\{0\}\right\}
$$

is a complete set of irreducible unitary representations of $H_{n}$.

Let $R$ be the quasi-regular representation of $H_{n}$ on $L^{2}\left(\Gamma_{r} \backslash H_{n}\right)$, i.e.,

$$
\left(R\left(\gamma^{\prime}\right) f\right)([\gamma])=f\left(\left[\gamma \gamma^{\prime}\right]\right)
$$


holds for all $f \in L^{2}\left(\Gamma_{r} \backslash H_{n}\right),[\gamma] \in \Gamma_{r} \backslash H_{n}$ and $\gamma \in H_{n}$. By 2, Lemma 3.7], $\left(R, L^{2}\left(\Gamma_{r} \backslash H_{n}\right)\right)$ decomposes

$$
\begin{aligned}
& \left(R, L^{2}\left(\Gamma_{r} \backslash H_{n}\right)\right) \\
\cong & \left(\bigoplus_{\tau \in \mathcal{A}_{r}}\left(f_{\tau}, \mathbb{C}\right)\right) \bigoplus\left(\bigoplus_{c \in \mathbb{Z} \backslash\{0\}}\left(\left|c^{n}\right| r_{1} \ldots r_{n}\right)\left(\pi_{c}, L^{2}\left(\mathbb{R}^{n}\right)\right)\right) .
\end{aligned}
$$

Finally, we remark on the Laplacian and Ricci curvature of a unimodular Lie group with a left invariant metric.

Lemma 4.3. Let $G$ be an $m$-dimensional unimodular Lie group with Lie algebra $\mathfrak{g}$, i.e., Trace $(a d(X))=0$ for all $X \in \mathfrak{g}$. Take a left invariant metric $g$ on $G$. Let $\left\{U_{i}\right\}$ be the $g$-orthonormal basis of $\mathfrak{g}$. Consider $\left\{U_{i}\right\}$ as left invariant vector fields on $G$.

(i) We have $\Delta_{g} f=-\sum_{i=1}^{m} U_{i}^{2} f$ for $f \in C^{\infty}(G)$,

(ii) We have

$$
\begin{aligned}
\operatorname{Ric}\left(U_{i}, U_{j}\right)= & -\frac{1}{2} \sum_{k=1}^{m} g\left(\left[U_{k}, U_{i}\right],\left[U_{k}, U_{j}\right]\right)-\frac{1}{2} \sum_{k=1}^{m} g\left(U_{k},\left[\left[U_{k}, U_{i}\right], U_{j}\right]\right) \\
& +\frac{1}{4} \sum_{k, l=1}^{m} g\left(U_{i},\left[U_{k}, U_{l}\right]\right) g\left(U_{j},\left[U_{k}, U_{l}\right]\right) .
\end{aligned}
$$

(iii) We have

$$
\nabla^{*} \operatorname{Ric}(\nabla f, \cdot)=-\sum_{i, j=1}^{m} \operatorname{Ric}\left(U_{i}, U_{j}\right) U_{i} U_{j} f
$$

for $f \in C^{\infty}(G)$.

Straight calculation implies the lemma. See [11, Corollary 1] for the proof of (i). Note that the Heisenberg groups is unimodular.

Now, we are in position to prove Proposition 4.2

Proof of Proposition 4.2. We can assume that $\Gamma=\Gamma_{r}$ and that $g$ is of the form

$$
g=\left[\begin{array}{cc}
h & 0 \\
0 & g_{n+1}
\end{array}\right] \text {. }
$$

By [2, Lemma 3.5], there exist an $h$-orthonormal basis $\left\{X_{1}^{\prime}, \cdots, X_{n}^{\prime}, Y_{1}^{\prime}, \cdots, Y_{n}^{\prime}\right\}$ of $\mathfrak{z}_{n}^{\perp}$ and positive constants $d_{1}, \cdots, d_{n}>0$ such that

$$
\left[X_{i}^{\prime}, X_{j}^{\prime}\right]=\left[Y_{i}^{\prime}, Y_{j}^{\prime}\right]=0, \quad\left[X_{i}^{\prime}, Y_{j}^{\prime}\right]=\delta_{i j} d_{i}^{2} Z
$$

for all $1 \leq i, j \leq n$. We put $Z^{\prime}=\left(g_{2 n+1}\right)^{-1 / 2} Z$. Then, $\left\{X_{1}^{\prime}, \cdots, X_{n}^{\prime}, Y_{1}^{\prime}, \cdots, Y_{n}^{\prime}, Z^{\prime}\right\}$ is a $g$-orthonormal basis of $\mathfrak{h}_{n}$, and $\left[X_{i}^{\prime}, Y_{i}^{\prime}\right]=d_{i}^{2} \sqrt{g_{2 n+1}} Z^{\prime}$ holds. By Lemma 4.3 (ii), we have

$$
\begin{aligned}
\operatorname{Ric}\left(X_{i}^{\prime}, X_{j}^{\prime}\right) & =\operatorname{Ric}\left(Y_{i}^{\prime}, Y_{j}^{\prime}\right)=-\frac{1}{2} \delta_{i j} d_{i}^{4} g_{2 n+1}, \\
\operatorname{Ric}\left(X_{i}^{\prime}, Y_{j}^{\prime}\right) & =\operatorname{Ric}\left(X_{i}^{\prime}, Z^{\prime}\right)=\operatorname{Ric}\left(Y_{i}^{\prime}, Z^{\prime}\right)=0, \\
\operatorname{Ric}\left(Z^{\prime}, Z^{\prime}\right) & =\frac{1}{2} \sum_{i=1}^{n} d_{i}^{4} g_{2 n+1},
\end{aligned}
$$

for all $1 \leq i, j \leq n$. Therefore, by Lemma 4.3 (iii), we have

$$
\nabla^{*}(\operatorname{Ric}(\nabla f, \cdot))=\frac{1}{2} \sum_{i=1}^{n} d_{i}^{4} g_{2 n+1}\left(\left(X_{i}^{\prime}\right)^{2}+\left(Y_{i}^{\prime}\right)^{2}\right) f-\frac{1}{2} \sum_{i=1}^{n} d_{i}^{4} g_{2 n+1}\left(Z^{\prime}\right)^{2} f
$$

for all $f \in C^{\infty}(M)$. 
Let $f \in L^{2}\left(\Gamma_{r} \backslash H_{n}\right)$ belongs to $\left(f_{\tau}, \mathbb{C}\right)$-component for some $\tau \in \mathcal{A}_{r}$. Take real numbers $a_{i}, b_{i} \in \mathbb{R}$ such that $\tau=\sum\left(a_{i}\left(X_{i}^{\prime}\right)^{*}+b_{i}\left(Y_{i}^{\prime}\right)^{*}\right)$, where $\left\{\left(X_{1}^{\prime}\right)^{*}, \ldots,\left(X_{n}^{\prime}\right)^{*}\right.$, $\left.\left(Y_{1}^{\prime}\right)^{*}, \ldots,\left(Y_{n}^{\prime}\right)^{*},\left(Z^{\prime}\right)^{*}\right\}$ denotes the dual basis of $\left\{X_{1}^{\prime}, \cdots, X_{n}^{\prime}, Y_{1}^{\prime}, \cdots, Y_{n}^{\prime}, Z^{\prime}\right\}$. Then, we have

$$
\begin{gathered}
\Delta f=4 \pi^{2} \sum_{i=1}^{n}\left(a_{i}^{2}+b_{i}^{2}\right) f \\
\nabla^{*}(\operatorname{Ric}(\nabla f, \cdot))=-\sum_{i=1}^{n} 2 \pi^{2} d_{i}^{4} g_{2 n+1}\left(a_{i}^{2}+b_{i}^{2}\right) f
\end{gathered}
$$

for all $f \in C^{\infty}(M)$. Therefore, we have

$$
-\frac{\sum_{i=1}^{n} 2 \pi^{2} d_{i}^{4} g_{2 n+1}\left(a_{i}^{2}+b_{i}^{2}\right)}{\left(4 \pi^{2} \sum_{i=1}^{n}\left(a_{i}^{2}+b_{i}^{2}\right)\right)^{2}} \Delta^{2} f=\nabla^{*}(\operatorname{Ric}(\nabla f, \cdot)) .
$$

We next consider the functions that belongs to $\left(\pi_{c}, L^{2}\left(\mathbb{R}^{n}\right)\right)$-component for some $c \in \mathbb{Z} \backslash\{0\}$. We consider the functions in $L^{2}\left(\mathbb{R}^{n}\right)$ as the functions in $L^{2}\left(\Gamma_{r} \backslash H_{n}\right)$. We define $\psi_{*}: \mathfrak{h}_{n} \rightarrow \mathfrak{h}_{n}$ by $\psi_{*}\left(X_{i}^{\prime}\right)=d_{i} X_{i}, \psi_{*}\left(Y_{i}^{\prime}\right)=d_{i} Y_{i}$ and $\psi_{*}(Z)=Z$. Then, $\psi_{*}$ is automorphism of $\mathfrak{h}_{n}$ and lifts to the automorphism $\psi: H_{n} \rightarrow H_{n}$. We put $\pi_{c}^{\prime}=\pi_{c} \circ \psi$. Then, $\pi_{c}^{\prime}$ is irreducible representation of $H_{n}$ on $L^{2}\left(\mathbb{R}^{n}\right)$, and $\pi_{c}^{\prime}(Z)=$ $\pi_{c}(Z)$. Thus, $\pi_{c}^{\prime}$ is unitary equivalent to $\pi_{c}$, and so there exists an isomorphism $\Psi: L^{2}\left(R^{n}\right) \rightarrow L^{2}\left(R^{n}\right)$ such that $\Psi\left(\pi_{c}(\gamma) f\right)=\pi_{c}^{\prime}(\gamma) \Psi(f)$ for all $f \in L^{2}\left(R^{n}\right)$ and $\gamma \in H_{n}$. We have $\left(\pi_{c}^{\prime}\right)_{*}\left(X_{i}^{\prime}\right)=d_{i}\left(\pi_{c}\right)_{*}\left(X_{i}\right),\left(\pi_{c}^{\prime}\right)_{*}\left(Y_{i}^{\prime}\right)=d_{i}\left(\pi_{c}\right)_{*}\left(Y_{i}\right)$ and $\left(\pi_{c}^{\prime}\right)_{*}\left(Z^{\prime}\right)=$ $\left(g_{2 n+1}\right)^{-1 / 2}\left(\pi_{c}\right)_{*}(Z)$. If $\Psi(f) \in \mathcal{S}\left(R^{n}\right)$ (where $\mathcal{S}\left(\mathbb{R}^{n}\right)$ denotes the Schwartz space), we have

$$
\begin{aligned}
& \Psi(\Delta f)(u) \\
= & \Psi\left(-\sum_{i=1}^{n}\left(\left(\left(\pi_{c}\right)_{*}\left(X_{i}^{\prime}\right)\right)^{2}+\left(\left(\pi_{c}\right)_{*}\left(Y_{i}^{\prime}\right)\right)^{2}\right) f-\left(\left(\pi_{c}\right)_{*}\left(Z^{\prime}\right)\right)^{2} f\right)(u) \\
= & {\left[-\sum_{i=1}^{n}\left(\left(\left(\pi_{c}^{\prime}\right)_{*}\left(X_{i}^{\prime}\right)\right)^{2}+\left(\left(\pi_{c}^{\prime}\right)_{*}\left(Y_{i}^{\prime}\right)\right)^{2}\right)-\left(\left(\pi_{c}^{\prime}\right)_{*}\left(Z^{\prime}\right)\right)^{2}\right] \Psi(f)(u) } \\
= & {\left[-\sum_{i=1}^{n} d_{i}^{2}\left(\left(\left(\pi_{c}\right)_{*}\left(X_{i}\right)\right)^{2}+\left(\left(\pi_{c}\right)_{*}\left(Y_{i}\right)\right)^{2}\right)-\frac{1}{g_{2 n+1}}\left(\left(\pi_{c}\right)_{*}(Z)\right)^{2}\right] \Psi(f)(u) } \\
= & {\left[\sum_{i=1}^{n} d_{i}^{2}\left(4 \pi^{2} c^{2} u_{i}^{2}-\frac{\partial^{2}}{\partial u_{i}^{2}}\right)+\frac{4 \pi^{2} c^{2}}{g_{2 n+1}}\right] \Psi(f)(u), } \\
& \Psi\left(\nabla^{*}(\operatorname{Ric}(\nabla f, \cdot))\right) \\
= & {\left[\frac{1}{2} \sum_{i=1}^{n} d_{i}^{6} g_{2 n+1}\left(\left(\left(\pi_{c}\right)_{*}\left(X_{i}\right)\right)^{2}+\left(\left(\pi_{c}\right)_{*}\left(Y_{i}\right)\right)^{2}\right)-\frac{1}{2} \sum_{i=1}^{n} d_{i}^{4}\left(\left(\pi_{c}\right)_{*}(Z)\right)^{2}\right] \Psi(f) } \\
= & {\left[-\frac{1}{2} \sum_{i=1}^{n} d_{i}^{6} g_{2 n+1}\left(4 \pi^{2} c^{2} u_{i}^{2}-\frac{\partial^{2}}{\partial u_{i}^{2}}\right)+2 \sum_{i=1}^{n} d_{i}^{4} \pi^{2} c^{2}\right] \Psi(f) . }
\end{aligned}
$$

For $k=\left(k_{1}, \ldots, k_{n}\right) \in \mathbb{Z}_{\geq 0}^{n}$, we put

$$
h_{k}(u)=\exp \left(|u|^{2} / 2\right) \partial^{k} \exp \left(-|u|^{2}\right) \quad\left(u \in \mathbb{R}^{n}\right),
$$

where

$$
\partial^{k}=\frac{\partial^{k_{1}+\cdots+k_{n}}}{\partial u_{1}^{k_{1}} \cdots \partial u_{n}^{k_{n}}}
$$


These functions are known as Hermite functions, and form a complete orthonormal system of $L^{2}\left(\mathbb{R}^{n}\right)$. We have

$$
\left(u_{i}^{2}-\frac{\partial^{2}}{\partial u_{i}^{2}}\right) h_{k}=\left(2 k_{i}+1\right) h_{k}
$$

for $1 \leq i \leq n$. Thus, for $\widetilde{h}_{k}(u)=h_{k}(\sqrt{2 \pi|c|} u)$, we have

$$
\left(4 \pi^{2} c^{2} u_{i}-\frac{\partial^{2}}{\partial u_{i}^{2}}\right) \widetilde{h}_{k}=2 \pi|c|\left(2 k_{i}+1\right) \widetilde{h}_{k} .
$$

Therefore, for $f_{k}=\Psi^{-1}\left(\widetilde{h}_{k}\right)$, we have

$$
\begin{aligned}
& \Psi\left(\Delta f_{k}\right)(u) \\
= & {\left[\frac{4 \pi^{2} c^{2}}{g_{2 n+1}}+\sum_{i=1}^{n} 2 \pi|c| d_{i}^{2}\left(2 k_{i}+1\right)\right] \Psi\left(f_{k}\right)(u), } \\
& \Psi\left(\nabla^{*}\left(\operatorname{Ric}\left(\nabla f_{k}, \cdot\right)\right)\right) \\
= & {\left[2 \pi^{2} c^{2} \sum_{i=1}^{n} d_{i}^{4}-\sum_{i=1}^{n} \pi|c| d_{i}^{6} g_{2 n+1}\left(2 k_{i}+1\right)\right] \Psi\left(f_{k}\right), }
\end{aligned}
$$

and so

$$
\frac{2 \pi^{2} c^{2} \sum_{i=1}^{n} d_{i}^{4}-\sum_{i=1}^{n} \pi|c| d_{i}^{6} g_{2 n+1}\left(2 k_{i}+1\right)}{\left[\frac{4 \pi^{2} c^{2}}{g_{2 n+1}}+\sum_{i=1}^{n} 2 \pi|c| d_{i}^{2}\left(2 k_{i}+1\right)\right]^{2}} \Delta^{2} f_{k}=\nabla^{*}\left(\operatorname{Ric}\left(\nabla f_{k}, \cdot\right)\right) .
$$

For $c \in \mathbb{Z} \backslash\{0\}, k \in \mathbb{Z}_{\geq 0}^{n}$, we put

$$
\Omega(c, k, g)=\frac{2 \pi^{2} c^{2} \sum_{i=1}^{n} d_{i}^{4}-\sum_{i=1}^{n} \pi|c| d_{i}^{6} g_{2 n+1}\left(2 k_{i}+1\right)}{\left[\frac{4 \pi^{2} c^{2}}{g_{2 n+1}}+\sum_{i=1}^{n} 2 \pi|c| d_{i}^{2}\left(2 k_{i}+1\right)\right]^{2}} .
$$

By (20) and (21), we have

$$
\Omega_{1}(g)=\sup \left\{\Omega(c, k, g): c \in \mathbb{Z}_{>0}, k \in \mathbb{Z}_{\geq 0}^{n}\right\} .
$$

If $\Omega(c, k, g)>0$, then we have $\Omega(c, 0, g) \geq \Omega(c, k, g)$. Thus, we have $\Omega_{1}(g)=$ $\sup \left\{\Omega(c, 0, g): c \in \mathbb{Z}_{>0}\right\}$. We have, for $c \in \mathbb{Z}_{>0}$,

$$
\Omega(c, 0, g)=\frac{g_{2 n+1}^{2}}{8 \pi^{2}} \frac{c \sum_{i=1}^{n} d_{i}^{4}-\frac{g_{2 n+1}}{2 \pi} \sum_{i=1}^{n} d_{i}^{6}}{c\left(c+\frac{g_{2 n+1}}{2 \pi} \sum_{i=1}^{n} d_{i}^{2}\right)^{2}} .
$$

Put $p=\sum_{i=1}^{n} d_{i}^{2}, q=\sum_{i=1}^{n} d_{i}^{4}$ and $r=\sum_{i=1}^{n} d_{i}^{6}$. For $x \in \mathbb{R}_{>0}$,

$$
F(x)=\frac{g_{2 n+1}^{2}}{8 \pi^{2}} \frac{q x-\frac{g_{2 n+1}}{2 \pi} r}{x\left(x+\frac{g_{2 n+1}}{2 \pi} p\right)^{2}}
$$

takes its maximum at $x=\frac{g_{2 n+1}}{2 \pi} \frac{r}{q}\left(\sqrt{\frac{9}{16}+\frac{p q}{2 r}}+\frac{3}{4}\right)$. Thus, we have

$$
\begin{aligned}
& \Omega(c, 0, g) \\
\leq & F\left(\frac{g_{2 n+1}}{2 \pi} \frac{r}{q}\left(\sqrt{\frac{9}{16}+\frac{p q}{2 r}}+\frac{3}{4}\right)\right) \\
= & \frac{1}{2} \frac{q^{3}}{r^{2}} \frac{\sqrt{\frac{9}{16}+\frac{p q}{2 r}}-\frac{1}{4}}{\left(\sqrt{\frac{9}{16}+\frac{p q}{2 r}}+\frac{3}{4}\right)\left(\frac{p q}{r}+\sqrt{\frac{9}{16}+\frac{p q}{2 r}}+\frac{3}{4}\right)^{2}}
\end{aligned}
$$

By the Cauchy-Schwartz inequality, we have

$$
q^{2}=\left(\sum_{i=1}^{n} d_{i}^{4}\right)^{2} \leq\left(\sum_{i=1}^{n} d_{i}^{2}\right)\left(\sum_{i=1}^{n} d_{i}^{6}\right)=p r
$$


and so

$$
\frac{q^{3}}{r^{2}} \leq \frac{p q}{r}
$$

Put $X=\sqrt{\frac{9}{16}+\frac{p q}{2 r}}$. Since $1 \leq \frac{p q}{r} \leq n$, we have $\sqrt{\frac{17}{16}} \leq X \leq \sqrt{\frac{9}{16}+\frac{n}{2}}$. We have $\frac{p q}{r}=2 X^{2}-\frac{9}{8}$. Therefore, we get

$$
\begin{aligned}
\Omega(c, 0, g) & \leq \frac{\left(X^{2}-\frac{9}{16}\right)\left(X-\frac{1}{4}\right)}{\left(X+\frac{3}{4}\right)\left(2 X^{2}+X-\frac{3}{8}\right)^{2}} \\
& =\frac{1}{4} \frac{X-\frac{3}{4}}{\left(X-\frac{1}{4}\right)\left(X+\frac{3}{4}\right)^{2}} .
\end{aligned}
$$

If $n \geq 2$, the right side of (22) takes its maximum at $X=\frac{5}{4}$, and so $\Omega(c, 0, g) \leq$ $\frac{1}{32}$. If we take a sequence of left invariant metrics $\{g(l)\}$ such that $d_{1}(l)=d_{2}(l)=$ constant, $\lim _{l \rightarrow \infty} d_{i}(l)=0(k \geq 3)$ and

$$
c(l)=\frac{g_{2 n+1}(l)}{2 \pi}\left(\frac{3 r(l)}{4 q(l)}+\sqrt{\frac{9}{16} \frac{r(l)^{2}}{q(l)^{2}}+\frac{p(l) r(l)}{2 q(l)}}\right) \in \mathbb{Z}_{>0},
$$

then $\lim _{l \rightarrow \infty} \Omega(c(l), 0, g(l))=\frac{1}{32}$. Thus, we get

$$
\sup _{g \in \mathrm{L}(M)} \Omega_{1}(g)=\frac{1}{32}
$$

If $n=1$, we have $X=\sqrt{\frac{17}{16}}$, and so

$$
\Omega(c, 0, g) \leq \frac{\sqrt{\frac{17}{16}}-\frac{3}{4}}{\left(\sqrt{\frac{17}{16}}-\frac{1}{4}\right)\left(\sqrt{\frac{17}{16}}+\frac{3}{4}\right)^{2}}=\frac{1}{16} \frac{\sqrt{17}-3}{(\sqrt{17}-1)(\sqrt{17}+3)^{2}} .
$$

If we take a left invariant metric such that

$$
c=\frac{g_{2 n+1}}{2 \pi}\left(\frac{3 r}{4 q}+\sqrt{\frac{9}{16} \frac{r^{2}}{q^{2}}+\frac{p r}{2 q}}\right) \in \mathbb{Z}_{>0},
$$

then we have $\Omega(c, 0, g)=\frac{1}{16} \frac{\sqrt{17}-3}{(\sqrt{17}-1)(\sqrt{17}+3)^{2}}$. Thus, we get

$$
\sup _{g \in \mathrm{L}(M)} \Omega_{1}(g)=\frac{1}{16} \frac{\sqrt{17}-3}{(\sqrt{17}-1)(\sqrt{17}+3)^{2}} .
$$

\section{Appendix A. The proof of Lemma 2.5}

In this appendix we prove Lemma 2.5

Proof of Lemma 2.5. Suppose that $S \leq 0$ does not hold. Then, there exist $x_{0} \in M$ and $X_{0} \in T_{x_{0}} M$ such that $S\left(X_{0}, X_{0}\right)>0$. We put $2 \delta=S\left(X_{0}, X_{0}\right)>0$. There exist constants $C_{1}, C_{2}>0$ and a local coordinate $\left(U ; x^{1}, x^{2}, \ldots, x^{n}\right)$ centered at $x_{0}$ such that

$$
\begin{aligned}
\left(\nabla x^{1}\right)_{x_{0}} & =X_{0}, \\
S\left(\nabla x^{1}, \nabla x^{1}\right) & \geq \delta, \\
C_{1} d \mu_{\mathbb{R}^{n}} \leq d \mu_{g} & \leq C_{2} d \mu_{\mathbb{R}^{n}} \quad \text { on } U .
\end{aligned}
$$


We take a positive integer $N \in \mathbb{N}$ such that $\left[-\frac{2 \pi}{N}, \frac{2 \pi}{N}\right]^{n} \subset U$ and take a smooth function $\psi: U \rightarrow \mathbb{R}$ such that

$$
\begin{aligned}
\operatorname{supp} \psi & \subset\left(-\frac{2 \pi}{N}, \frac{2 \pi}{N}\right)^{n}, \\
\psi & =1 \text { on }\left[-\frac{\pi}{N}, \frac{\pi}{N}\right]^{n}, \\
0 \leq \psi & \leq 1 \quad \text { on } U .
\end{aligned}
$$

Since $\psi$ has compact support, we can take a constant $C_{3}>0$ such that

$$
\begin{aligned}
|S(\nabla \psi, \nabla \psi)| & \leq C_{3}, \\
\left|S\left(\nabla \psi, \nabla x^{1}\right)\right| & \leq C_{3} \text { on } U .
\end{aligned}
$$

For each positive integer $m \in \mathbb{N}$, we define a function $u_{m}:\left[-\frac{2 \pi}{N}, \frac{2 \pi}{N}\right]^{n} \rightarrow \mathbb{R}$ by

$$
u_{m}(x)=\sin \left(m N x^{1}\right) .
$$

We regard $\psi u_{m}$ as a smooth function on $M: \psi u_{m} \in C^{\infty}(M)$. For each $k, K \in \mathbb{N}$ we define a $k$-dimensional subspace $V_{k, K} \subset C^{\infty}(M)$ by

$$
V_{k, K}=\operatorname{Span}_{\mathbb{R}}\left\{\psi u_{K+1}, \cdots, \psi u_{K+k}\right\} .
$$

Take $a=\left(a^{K+1}, \cdots, a^{k+K}\right) \in \mathbb{R}^{K}$ and define $|a|^{2}=\left(a^{K+1}\right)^{2}+\cdots+\left(a^{K+k}\right)^{2}$. In $\mathrm{U}$, we have

$$
\begin{aligned}
& S\left(\nabla\left(\sum_{i=K+1}^{K+k} \psi a^{i} u_{i}\right), \nabla\left(\sum_{j=K+1}^{K+k} \psi a^{j} u_{j}\right)\right) \\
& =\sum_{i, j=K+1}^{K+k} a^{i} a^{j}\left\{\sin \left(i N x^{1}\right) \sin \left(j N x^{1}\right) S(\nabla \psi, \nabla \psi)\right. \\
& \quad+\left(j N \psi \sin \left(i N x^{1}\right) \cos \left(j N x^{1}\right)+i N \psi \sin \left(j N x^{1}\right) \cos \left(i N x^{1}\right)\right) S\left(\nabla \psi, \nabla x^{1}\right) \\
& \left.\quad+i j N^{2} \psi^{2} \cos \left(i N x^{1}\right) \cos \left(j N x^{1}\right) S\left(\nabla x^{1}, \nabla x^{1}\right)\right\}
\end{aligned}
$$

Since $|\sin | \leq 1$ and $|\cos | \leq 1$,

(the left hand side of (23)

$$
\begin{aligned}
& \geq-\sum_{i, j=K+1}^{K+k}\left(\left|a^{i}\right|\left|a^{j}\right| C_{3}(1+i N+j N)\right) \\
& \quad+\left(\sum_{i=K+1}^{K+k} i N a^{i} \psi \cos \left(i N x^{1}\right)\right)^{2} S\left(\nabla x^{1}, \nabla x^{1}\right) \\
& \geq-3 k(K+k) N|a|^{2} C_{3} \\
& \quad+\left(\sum_{i=K+1}^{K+k} i N a^{i} \psi \cos \left(i N x^{1}\right)\right)^{2} S\left(\nabla x^{1}, \nabla x^{1}\right) \\
& \geq-3 k(K+k) N|a|^{2} C_{3}+\left(\sum_{i=K+1}^{K+k} i N a^{i} \psi \cos \left(i N x^{1}\right)\right)^{2} \delta
\end{aligned}
$$


By integrate both sides of (24), we have

$$
\begin{aligned}
& \int_{M} S\left(\nabla\left(\sum_{i=K+1}^{K+k} \psi a^{i} u_{i}\right), \nabla\left(\sum_{j=K+1}^{K+k} \psi a^{j} u_{j}\right)\right) d \mu_{g} \\
& \geq \int_{\left[-\frac{2 \pi}{N}, \frac{2 \pi}{N}\right]^{n}}\left(-3 k(K+k) N|a|^{2} C_{3}+\left(\sum_{i=K+1}^{K+k} i N a^{i} \psi \cos \left(i N x^{1}\right)\right)^{2} \delta\right) d \mu_{g} \\
& \geq-\int_{\left[-\frac{2 \pi}{N}, \frac{2 \pi}{N}\right]^{n}} 3 k(K+k) N|a|^{2} C_{2} C_{3} d \mu_{\mathbb{R}} \\
&+\int_{\left[-\frac{\pi}{N}, \frac{\pi}{N}\right]^{n}}\left(\sum_{i=K+1}^{K+k} i N a^{i} \cos \left(i N x^{1}\right)\right)^{2} \delta C_{1} d \mu_{\mathbb{R}} \\
&= 3 k(K+k) N|a|^{2} C_{2} C_{3}\left(\frac{4 \pi}{N}\right)^{n} \\
&+\sum_{i, j=K+1}^{K+k} i j N^{2} a^{i} a^{j} \delta C_{1}\left(\frac{2 \pi}{N}\right)^{n-1} \int_{-\frac{\pi}{N}}^{\frac{\pi}{N}} \cos \left(i N x^{1}\right) \cos \left(j N x^{1}\right) d x^{1} \\
&= 3 k(K+k) N|a|^{2} C_{2} C_{3}\left(\frac{4 \pi}{N}\right)^{n}+\sum_{i, j=K+1}^{K+k} i j N^{2} a^{i} a^{j} \delta C_{1}\left(\frac{2 \pi}{N}\right)^{n-1} \frac{\pi}{N} \delta_{i j} \\
& \geq|a|^{2}\left(-3 k(K+k) N C_{2} C_{3}\left(\frac{4 \pi}{N}\right)^{n}+K^{2} N^{2} \delta C_{1}\left(\frac{2 \pi}{N}\right)^{n-1} \frac{\pi}{N}\right) .
\end{aligned}
$$

For any $k \in \mathbb{N}$ there exists a positive integer $K \in N$ such that

$$
-3 k(K+k) N C_{2} C_{3}\left(\frac{4 \pi}{N}\right)^{n}+K^{2} N^{2} \delta C_{1}\left(\frac{2 \pi}{N}\right)^{n-1} \frac{\pi}{N}>0,
$$

and so we have $\int_{M} S(\nabla v, \nabla v) d \mu_{g}>0$ for any $v \in V_{k, K} \backslash\{0\}$ by (25).

We define $V_{k}$ as the image of the linear map $V_{k, K} \rightarrow C^{\infty}(M) \cap H, v \mapsto v-$ $\frac{1}{\operatorname{Vol}(M)} \int_{M} v d \mu_{g}$. Since the Kernel is $\{0\}, V_{k}$ is $k$-dimensional. Moreover, for any $v \in V_{k} \backslash\{0\}$, we have $\int_{M} S(\nabla v, \nabla v) d \mu_{g}>0$; therefore $\Lambda_{S}(v)>0$.

\section{REFERENCES}

[1] R. L. Bishop, B. O'Neill, Manifolds of Negative curvature, Trans. Amer. Math. Soc. 145 (1969), 1-49.

[2] C. S. Gordon, E. N. Wilson, The spectrum of the Laplacian on Riemannian Heisenberg manifolds, Michigan Math. J. 33 (1986), 253-271.

[3] J. F. Grosjean, A new Lichnerowicz-Obata estimate in the presence of a parallel p-form, Manuscripta Math. 107 (2002), no. 4, 503-520.

[4] W. Kühnel, Conformal transformations between Einstein spaces, Conformal geometry (Bonn, 1985/1986), Aspects Math., E12, vieweg, Braunschweig (1988), 105-146.

[5] A. Lichnerowicz, Géométrie des groupes de transformations, Travaux et Recherches Mathématiques, III. Dunod, Paris (1958).

[6] M. Obata, Certain conditions for a Riemannian manifold to be isometric with a sphere, J. Math. Soc. Japan 14 (1962), 333-340.

[7] M. Sugiura, Fourier series of smooth functions on compact Lie groups, Osaka J. Math. 8 (1971), 33-47.

[8] Y. Tashiro, Complete Riemannian manifolds and some vector fields, Trans. Amer. Math. Soc. 117, (1965) 251-275.

[9] Y. Tashiro, Conformal transformations in complete Riemannian manifolds, Publ. the Study Group of Geometry, Vol. 3 (1967).

[10] S. Tanno, Eigenvalues of the Laplacian of Riemannian manifolds, Tôhoku Math. J. (2) 25, (1973), 391-403.

[11] H. Urakawa, On the least positive eigenvalue of the Laplacian for compact group manifolds, J. math Soc .Japan 31, No. 1 (1979), 209-226. 
ROTATIONAL SYMMETRY OF THE STANDARD SPHERE

Graduate School of Mathematics, Nagoya University, Chikusa-Ku Nagoya, 464-8602, JAPAN

E-mail address: m16100c@math.nagoya-u.ac.jp 